\title{
Capítulo I \\ La Apuesta por una Educación Propia en los Alrededores del Quilotoa
}

Sebastián Granda Merchán

\section{SciELO Books / SciELO Livros / SciELO Libros}

GRANDA MERCHÁN, S. La Apuesta por una Educación Propia en los Alrededores del Quilotoa. In: Estado, educación y pueblos indígenas en los Andes ecuatorianos: la experiencia del Sistema de Escuelas Indígenas de Cotopaxi [online]. Quito: Editorial Abya-Yala, 2020, pp. 35-87. Investigación Decolonial series, n. 2. ISBN: 978-9978-10-457-6.

http://doi.org/10.7476/9789978105733.0003.

\section{(C) (D)}

All the contents of this work, except where otherwise noted, is licensed under a Creative Commons Attribution 4.0 International license.

Todo o conteúdo deste trabalho, exceto quando houver ressalva, é publicado sob a licença Creative Commons Atribição 4.0.

Todo el contenido de esta obra, excepto donde se indique lo contrario, está bajo licencia de la licencia Creative Commons Reconocimento 4.0. 


\section{Capítulo I}

\section{LA APUESTA POR UNA EDUCACIÓN PROPIA EN LOS ALREDEDORES DEL QUILOTOA}

A partir de 1975, las comunidades indígenas de los páramos occidentales de la provincia de Cotopaxi, conjuntamente con el equipo misionero de Zumbahua, impulsaron un proyecto educativo al margen del Estado que buscó dar respuesta a las demandas de formación de su población. Nos referimos a aquella iniciativa que, en las décadas de los 70 y 80, se conoció con el nombre de Escuelas Indígenas del Quilotoa y que, desde 1988 en adelante, llevó el nombre de SEIC.

Este capítulo tiene como propósito central reconstruir la experiencia de las Escuelas Indígenas del Quilotoa, desde su creación en 1975 hasta 1988, año en el que las escuelas fueron reconocidas por el Estado ecuatoriano. Para lograr lo anterior, trabajaremos en dos frentes: por un lado, analizaremos su creación durante las décadas de los 70 y 80, teniendo como telón de fondo los procesos económicos, políticos y sociales que se vivían en la zona en cuestión; y, por otro, discutiremos la propuesta educativa y modelo de gestión que dichas escuelas pusieron en juego, así como el papel que desempeñaron en el marco del proyecto político impulsado por las comunidades indígenas.

Proponemos que las Escuelas Indígenas del Quilotoa surgieron como respuesta y al calor de las luchas impulsadas por las comunidades indígenas de la zona por dignificar su vida. Sostenemos también que dichas escuelas, a pesar de sus limitaciones logísticas y de financiamiento (propias de una iniciativa sostenida por las mismas comunidades), 
atendieron de una manera harto creativa sus necesidades educativas y contribuyeron, de esta manera, a alimentar su proyecto político.

Para el desarrollo del capítulo se revisaron fuentes primarias y secundarias, y se realizaron entrevistas a actores clave. Respecto a las fuentes primarias, se revisó la documentación existente en el archivo de la Inspectoría Salesiana, así como la que se encuentra en el Centro de Documentación del SEIC, con sede en Latacunga. En dicho centro reposan, entre otros documentos, las tesis de los primeros educadores comunitarios de la zona en las que se sistematiza y relata su experiencia de vida y la historia de las escuelas indígenas.

Con relación a las fuentes secundarias, se revisaron todas aquellas investigaciones y evaluaciones realizadas sobre las Escuelas Indígenas del Quilotoa, así como textos académicos que han analizado y discutido experiencias educativas similares desarrolladas dentro y fuera del país. Respecto al primer grupo de documentos, vale destacar que tuvimos acceso al informe de evaluación que el equipo de las escuelas indígenas realizó en 1988 sobre su trabajo y aporte a las comunidades, informe de un valor único por la abundancia de testimonios de los educadores comunitarios.

En cuanto a las entrevistas, se las hizo a actores clave que participaron de manera directa en la gestión de las escuelas y en el trabajo del día a día del aula. En este sentido, cabe destacar que tuvimos la oportunidad de dialogar, entre otros, con el padre José Manangón, sacerdote que ha liderado la experiencia educativa por más de treinta años, así como con seis de los educadores comunitarios más antiguos, los que iniciaron con la experiencia en la década de los 70 .

Hemos organizado el capítulo en cuatro partes. En la primera se hace un rápido repaso de la situación que se vivía en las zonas de Zumbahua y Chugchilán en las décadas de los 70 y 80, en las que se crearon los primeros centros de alfabetización y las primeras escuelas indígenas. En la segunda parte se discute su proceso de creación, poniendo énfasis en las motivaciones subjetivas que dieron lugar a esta. En la tercera se analizan las características centrales del proyecto educativo y el modelo de gestión implementados por las escuelas para atender a las demandas educativas 
de la población indígena. Y, en la última, se valora el papel que cumplieron las escuelas en el marco del proyecto político impulsado en la zona.

\section{Contexto en el que emergen las escuelas indígenas}

Para comprender el proceso de emergencia de las escuelas indígenas en las zonas aledañas a la laguna del Quilotoa, en primer lugar, es necesario entender la situación que vivía su población en las décadas de los 70 y 80 , y los procesos en los que aquella se encontraba envuelta, pues las escuelas surgieron y se consolidaron al calor de dichos procesos.

Las comunidades en la que se crearon las primeras escuelas indígenas se encuentran ubicadas en la zona occidental de la provincia de Cotopaxi, en aquellos territorios que pertenecen a las parroquias rurales de Zumbahua y Chugchilán ${ }^{6}$ que, a su vez, forman parte de lo que actualmente constituyen los cantones de Pujilí y Sigchos, respectivamente. ${ }^{7}$ Entre las comunidades indígenas que destacan por ser las pioneras en la creación de escuelas constan las siguientes: Guayama, Pilapuchín, Cuisana, Chaupi, Guantugloma, Quindigüa, Sarahuasi, Sarahugsha, Vaquería e Iracunga.

En términos agroecológicos se trata de una zona irregular, que va desde los 2500 hasta los 4500 metros de altura, y en la que la temperatura fluctúa entre los $3{ }^{\circ} \mathrm{C}$ y $15{ }^{\circ} \mathrm{C}$. La altura, la temperatura y otros factores - como las continuas heladas y los fuertes vientos - han hecho que esta zona (sobre todo la de altura) no sea muy propicia para la producción agrícola. De allí que el mismo Estado así como varias oenegés que han trabajado en la zona hayan sugerido, desde tiempo atrás, la producción silvipastoril como la más adecuada para el sector (Guerrero 1992).

Desde el punto de vista étnico-demográfico se trata de una zona que, para la década de los 70, estaba poblada fundamentalmente por

6 Con el paso de los años, la experiencia de las escuelas indígenas se amplió hacia: Panyatug, zona montañosa de la parroquia El Corazón (cantón Pangua); la zona conocida como El Bajío, ubicada en los cantones Pujilí y Saquisilí, y la parroquia de Pucayacu perteneciente al cantón La Maná.

7 El cantón Sigchos se creó el 21 de julio de 1992. Antes de su creación, Chugchilán pertenecía al cantón Pujilí, al igual que la parroquia de Zumbahua. 
población indígena kichwa. De acuerdo con un estudio de José Sánchez Parga (1986), para 1974 el porcentaje de población indígena en la zona llegaba a $90 \%$ a lo que habría que añadir que, en las comunidades rurales en las que se impulsaron las escuelas indígenas, el porcentaje de población indígena llegaba al $100 \%$, pues la población blanco-mestiza se concentraba, exclusivamente, en los centros parroquiales.

En términos socioeconómicos y políticos, podemos decir que la población indígena de la zona en cuestión vivía una situación compleja y aparentemente contradictoria durante las décadas de los 70 y 80. Mientras desde el punto de vista social y económico las comunidades indígenas vivían una situación de penuria, desde el punto de vista político vivían un importante proceso de activación política y organización.

En torno a la situación social, en las comunidades indígenas se vivía una situación de marcada pobreza como consecuencia de los largos e intensos procesos de explotación impulsados por los terratenientes y comerciantes de la zona, con el apoyo de las tenencias políticas y la Iglesia. ${ }^{8}$ Revisemos la manera en que el padre Felipe Mayordomo, uno de sacerdotes salesianos que trabajó en el sector durante la década de los 70 , describe la situación social de la población de la zona en una entrevista que le realizaran en Europa:

Entrevistador: Y cuando en 1971 llegasteis a Zumbagua, ¿cuál era la situación?

Padre Felipe Mayordomo: Imagínate: no había ni un miserable centro de salud. No había luz eléctrica, y sigue sin haberla. La tasa de analfabetismo estaba más alta que en Cotopaxi, rondaba el 80 por 100, y entre las mujeres, más alta todavía: el 90 por 100. La mortalidad, ese índice que aquí, en Europa, se mide en tantos por mil, allí llega al 60 por 100. Sí, créetelo.

Entrevistador: ¿O sea, que más de la mitad de los niños mueren antes de cumplir los dos años?

8 Las zonas rurales de la provincia de Cotopaxi, y en especial aquellas en las que se ha concentrado la población indígena, han sido las zonas, a nivel nacional, con mayores niveles de pobreza y extrema pobreza durante las últimas décadas (Sánchez Parga 2002). 
Padre Felipe Mayordomo: Eso es. Mira, un dato concreto que corrobora lo que dicen las estadísticas: el sacristán de Guangaje, localidad que está a 3800 metros de altura sobre el nivel del mar, tiene 13 hijos, solo le viven 5; los 8 restantes están en el cementerio.

Entrevistador: ¿Cuál es la esperanza de vida por aquellas alturas?

Padre Felipe Mayordomo: La media de vida solo llega a los 37 años, 40 menos que en España, ¿no? (Zuñeda s. f., 13)

A lo anterior hay que añadir la situación precaria de la agricultura y ganadería, principales actividades económicas de la zona en la década de los 70. En el proyecto que la Misión Salesiana preparó para intervenir en la zona se describe de la siguiente manera la situación de la agricultura en Zumbahua y Guangaje:

En cuanto al uso y destino de los recursos territoriales, la zona tiene estas posibilidades: tierras de páramo: 55,8 \%, tierras de labranza: 39,6\%, tierras estériles: $45 \%$.

La agricultura es arcaica y primitiva. Las técnicas empleadas son tradicionales debido a que son núcleos de población indígena que, al independizarse de las haciendas, constituyeron áreas de refugio de difícil penetración.

La mayor parte de las tierras han sido intensamente empleadas en cultivos ocasionales de patatas de las más variadas clases: ocas, mellocos, mashuas, habas y cebada, productos que son la base de la alimentación de los indígenas de esta área. (Comunidad Salesiana del Ecuador s. f., 15)

De igual manera, se describe la situación de la ganadería de la zona en los siguientes términos:

La zona de Zumbagua-Guangaje cuenta con una población ovina de más de 80000 cabezas, lo que corresponde a un $3 \%$ de la población ovina nacional.

Los indígenas son los que se dedican principalmente a la crianza de ovejas, manteniendo rebaños de un número que varía entre 20 y 200 cabezas que las pastorean en los terrenos comunales de los páramos.

La mayoría de las ovejas son criollas y el rendimiento de lana apenas llega a dos libras anuales por cabeza. Además, la calidad de la lana es mala. El borrego adulto de dos años apenas pesa cincuenta libras. La calidad de la carne igualmente es deficiente, en cuanto el porcentaje de proteínas es 
escaso, ya que los productos no proporcionan, en la alimentación, sales minerales y dietas complementarias.

El método de crianza es totalmente tradicional. No se aplican métodos selectivos para el mejoramiento de la raza, lo que ocasiona la degeneración sucesiva de los animales. (Comunidad Salesiana del Ecuador s. f., 16)

Situación similar vivían las comunidades indígenas de Chugchilán, aunque con un punto más en contra: a diferencia de la parroquia Zumbahua, en la que la hacienda se había disuelto en 1964 como resultado de la Reforma Agraria, la mayor parte de comunidades indígenas de Chugchilán seguían todavía sujetas a las haciendas de la zona, pues el proceso de recuperación de las tierras de las haciendas en dicho sector recién inició en la década de los 80 (Cicero 2014).

La falta de tierra, en algunos casos, su mala calidad, en otros, y los bajos niveles de productividad constituyen los factores que explican los problemas serios que las comunidades indígenas, tanto de Zumbahua como de Chugchilán, tenían que sortear en el día a día para cubrir sus necesidades básicas. Incluso explica las razones por las que la migración se convirtió en una estrategia de sobrevivencia para dichas comunidades. Veamos la magnitud de dicho fenómeno: en un estudio realizado por José Sánchez Parga (1991) en el sector de nuestro estudio, se aplicaron encuestas a una muestra de pobladores de Pilapuchín, comunidad de la parroquia Chugchilán. Del total de encuestados, el 73,33 \% afirmó que salía de manera regular a trabajar en Quevedo, Quito y Latacunga, como cargadores o albañiles, principalmente. La situación era similar en las demás comunidades.

Con respecto al segundo proceso, durante las décadas de los 70 y 80 , se vivía en la zona un intenso proceso de activación política de la población indígena, proceso que tenía como uno de los temas prioritarios la lucha por la recuperación de la tierra. Como adelantamos arriba, la situación de la tenencia de la tierra variaba en dicha década: mientras en la parroquia de Zumbahua se había desmantelado ya la hacienda, en la parroquia de Chugchilán el sistema aún seguía vivo.

Desde inicios del siglo XX, la zona de lo que actualmente es la parroquia de Zumbahua formaba parte de una gran hacienda que pertenecía 
a la Junta de Asistencia Social. Fruto de las luchas por la tierra desatadas en la zona desde 1940, con apoyo de la FEI, y por efecto de la Reforma Agraria implementada en 1965, la gran hacienda se disolvió y las tierras fueron distribuidas entre los exhuasipungueros, los yanaperos y los ocupantes arbitrarios (Martínez 2004) (Guerrero 1992).

En la parroquia de Chugchilán, en cambio, la lucha por la tierra inició recién en la década de los $70 .{ }^{9}$ Este fue el caso, por ejemplo, de la hacienda de Guayama en la que el proceso de lucha por la tierra inició en 1973 y concluyó en 1980, luego de siete años de trámites burocráticos y fuertes enfrentamientos con los dueños de la hacienda (Díaz 1997). Algo similar ocurrió en Chinaló, zona en la que también existía una gran hacienda y en la que los comuneros lograron recuperar las tierras en 1985 con intermediación del Instituto de Reforma Agraria y Colonización (IERAC), o en San Miguel de Pilapuchín, donde los comuneros accedieron a las tierras de la hacienda en 1988, mediante su compra. ${ }^{10}$

De acuerdo con Guerrero (1992), la lucha por la tierra en la zona contribuyó a fortalecer la organización y dio lugar a un proceso de reinvención y afirmación identitaria de la población indígena, procesos que posibilitaron la definición e implementación de una suerte de proyecto de desarrollo endógeno orientado a mejorar y dignificar sus condiciones de vida. A decir del mismo Guerrero:

A partir de la lucha por la tierra, se inicia todo un proceso que impulsa un mayor desarrollo organizativo, así como de maduración de su conciencia étnica. La ruptura de la hacienda [...] posibilita la afirmación de una identidad diferenciada que les conduce a la búsqueda de un proyecto para su "autodesarrollo", a fin de enfrentar su situación de marginalidad social y económica. (41)

Entre los componentes más relevantes del proyecto de "autodesarrollo" impulsado por la población indígena, destacan el componente

9 De acuerdo con Joselino Ante, líder indígena de la comunidad de Guayama, en la década de los 70 existían siete haciendas en la parroquia de Chugchilán (Tibán, Ilaquiche y Alfaro 2003).

10 Pedro Cunuhay, entrevista personal, 7 de mayo de 2014, comunidad de Chinaló, parroquia de Chugchilán, cantón Sigchos. 
productivo, pero asimismo los componentes educativo, político y simbólico. Pasemos a revisar, de manera breve, y de la mano de Guerrero, ${ }^{11}$ la naturaleza de estos componentes. El componente educativo lo analizaremos posteriormente con detenimiento.

En el ámbito productivo, a partir de la década de los 70, se impulsó en la zona un interesante proyecto para controlar los procesos de comercialización de los productos agrícolas generados por las comunidades indígenas. En un inicio el proyecto consistió en crear pequeñas tiendas comunales, pero luego, en 1979, se creó una tienda comunal grande para la comercialización directa de los productos. Así nació, en 1986, el sistema de comercialización conocido como Maquita Cushunchic (Comercializando como hermanos, en español).

Dicho sistema de comercialización permitió no solo cortar la dependencia que la población indígena tenía con los antiguos intermediarios, sino también impulsar, con una parte los excedentes del proceso de comercialización, varios de los proyectos demandados en la zona: implementación de infraestructura básica, construcción de molinos y apoyo a los ancianos y huérfanos, entre otros. Con el tiempo, el sistema de comercialización desbordó los márgenes del territorio en el que nació y, de manera paulatina, se fue ampliando hacia otras parroquias y provincias, llegando en pocos años a ciudades grandes como Quito.

En el ámbito político, destacó el proceso de reconfiguración del mapa de poder en la zona, como resultado del acceso de la población indígena a los espacios de poder local. Con el resquebrajamiento del poder local en la zona, por efecto de la disolución del sistema de hacienda, se dio un importante proceso de fortalecimiento de los cabildos, antigua instancia de poder de la comunidad pero, además, de acceso a la participación y control de las tenencias políticas, aparatos políticos-administrativos de las parroquias.

11 En su libro El saber del mundo de los cóndores: Identidad e insurgencia de la cultura andina, Patricio Guerrero reconstruye cada uno de los componentes del proyecto de autodesarrollo impulsado por las comunidades indígenas de la zona. Nos basamos en su texto para el desarrollo de las siguientes partes. 
El acceso de la población indígena a los espacios de poder local fue de gran importancia, pues facilitó, aunque no siempre con la organicidad esperada, el trabajo mancomunado entre las organizaciones indígenas, los cabildos y las tenencias políticas, orientado a promover "el control de las actividades especulativas de los comerciantes, impulsar y ampliar actividades de desarrollo, controlar el manejo de recursos que organismos de 'desarrollo' hacen en la región, para que no vayan a beneficiarse únicamente ciertos dirigentes o familias como ha ocurrido muchas veces" (Guerrero 1992, 110).

A nivel simbólico, destacan varios procesos que, si bien a primera vista no tienen mucho sentido, son de una importancia clave si se los mira en contexto. Nos referimos a procesos como la resignificación del compadrazgo, la recuperación de la fiesta, la resignificación del priostazgo y el control de la feria.

Con relación al primer tema, las comunidades indígenas se orientaron a recuperar la función simbólica del compadrazgo para que este se adscribiera al fortalecimiento de la relaciones de parentesco al interior de estas, y ya no a la búsqueda de prestigio y mejoramiento de las condiciones de vida en términos individuales: en la época de la hacienda, las familias indígenas ganaban prestigio y mejoraban sus condiciones de vida cuando hacían compadre al patrón de la hacienda o a uno de sus mayordomos.

En cuanto al segundo proceso, se constata en las comunidades de la zona un esfuerzo por recuperar el control de la fiesta y su papel en las comunidades. A diferencia del tiempo de la hacienda en el que la fiesta era realizada en el espacio de la hacienda y por voluntad y a gusto del patrón, aquella se desplaza al espacio comunal, y es la comunidad, en pleno, la que decide cómo, cuándo y por qué hacerla.

Conectado con el punto anterior está el tema del priostazgo. Con la disolución de la hacienda la figura del priostazgo se resignificó radicalmente, pues dejó de ser una estrategia más de control del patrón (el hacendado era el que nombraba al prioste y el que se beneficiaba por los préstamos en los que el prioste tenía que incurrir), y se convirtió en una figura que contribuyó a que la comunidad afirmara su poder y protagonismo: todos los 
comuneros eran priostes y, en calidad de tales, todos tenían la obligación de organizar y sostener la fiesta.

Y, sobre el tercer punto, se activó el interés por recuperar el control de la feria, espacio de intercambio de suprema importancia en la zona en cuestión y el mundo andino en general. Decimos recuperar el control de la feria, puesto que la apuesta de las organizaciones indígenas era retomar el control de varias de las dimensiones de la feria que, con el tiempo, habían perdido: el cobro de los impuestos, la regulación del funcionamiento de la feria en su conjunto, la regulación de los pesos y medidas que utilizaban los comerciantes mestizos y la regulación del tanque de agua y del camal para el desposte de los animales, entre otros, dimensiones que, con el tiempo, pasaron a manos de las organizaciones indígenas.

De todo lo anteriormente dicho, podemos concluir que, si bien la situación de vida no era fácil para las comunidades indígenas del sector, estas se encontraban en un interesante proceso político que tenía como finalidad central la recuperación del control de aquellos elementos clave para su desarrollo como pueblo. La apuesta por la construcción de un modelo de autodesarrollo, en palabras de Patricio Guerrero, o un modelo de etnodesarrollo, en la línea de Bonfil Batalla (1995). ${ }^{12}$

Fue en el marco y al calor de dicho proceso político que nacieron las Escuelas Indígenas del Quilotoa, y fue al fortalecimiento de dicho proceso que aquellas dirigieron toda su energía.

\section{El proceso de creación de las Escuelas Indígenas del Quilotoa}

\section{Actores involucrados}

Dos actores clave en la creación de las escuelas indígenas fueron la Comunidad Salesiana y los líderes de las comunidades indígenas de la zona. De allí que conviene dedicar unas líneas a cada uno de ellos y evidenciar su perspectiva con respecto a la educación.

12 Para Bonfil Batalla, por etnodesarrollo se entiende "el ejercicio de la capacidad social de un pueblo para construir su futuro, aprovechando para ello las enseñanzas de su experiencia histórica y los recursos reales y potenciales de su cultura, de acuerdo con un proyecto que se defina según sus propios valores y aspiraciones" (467). 
Los salesianos llegaron a Zumbahua en 1971, luego de que la Diócesis de Latacunga aceptara su petición de trabajar con la población kichwa de la zona occidental de la provincia de Cotopaxi. El 28 de junio de 1971 el Consejo de Presbiterio, reunido por pedido del obispo de Latacunga, demarcó el área misionera confiada a la Comunidad Salesiana, la cual incluía varias comunidades de altura ubicadas en territorios que actualmente constituyen las parroquias de Zumbahua y Guangaje. ${ }^{13} \mathrm{El}$ siguiente día, 29 de junio, se creó la parroquia eclesiástica de San Cruz de Zumbahua-Guangaje, con sede principal en el poblado de Zumbahua, y desde ese mismo día inició el trabajo de los salesianos en la zona (Guerrero y Creamer 1997).

Los salesianos entraron a la zona con el objetivo de impulsar un proyecto de desarrollo integral de la población indígena de la localidad. Mediante dicho proyecto los salesianos buscaban enfrentar problemáticas relacionadas con el ámbito productivo de los campesinos indígenas de la zona, su calidad de vida (salud y educación), el proceso organizativo de las comunidades y su vida espiritual. Así, el proyecto contemplaba las siguientes once líneas de trabajo: vías de comunicación, energía y riego, agricultura, ganadería, forestación, artesanía, salud, educación, organización de la comunidad, atención a los emigrantes y pastoral (Comunidad Salesiana del Ecuador s. f.).

Como consta en el párrafo anterior, el ámbito educativo fue contemplado, desde un inicio, como una línea de trabajo prioritaria dentro del proyecto de promoción de la población indígena. Desde el punto de vista de los salesianos, el problema con la educación impartida en la zona era, fundamentalmente, que desconocía la realidad de las comunidades indígenas y que, en tal medida, terminaba provocando efectos negativos en ellas. En el proyecto se definió la problemática de la siguiente manera:

La educación actual del indígena, con ciertos patrones formales de instrucción, impuestos por el Ministerio de Educación, ajenos al medio cultural del niño campesino, son acciones foráneas que no respetan el hecho cultural, menos social.

13 La parroquia civil de Zumbahua fue creada en 1972, un año después de la llegada de los salesianos a la zona. 
En la práctica, existe un colonialismo educativo, tratando de "civilizar" mediante patrones culturales extraños. La educación rural indígena actual es sobreañadida, desculturizadora y formal, creando incompatibilidad entre el trabajo del campo y el horario escolar. (Comunidad Salesiana del Ecuador s. f., 20)

Con base en dicho diagnóstico, los salesianos propusieron crear "una escuela nueva rural que defienda y desarrolle la cultura existente en el medio indígena y lograr así la integración a la cultura nacional" (Comunidad Salesiana del Ecuador s. f.). La escuela nueva debía repensar y cambiar los fundamentos y bases pedagógicas de la educación tradicional, y debía considerar tanto el tema de la lengua materna de la población (el kichwa), como la educación informal del hogar y el medio. En la misma línea, planteaban que el proyecto educativo debía alimentar el proyecto productivo de la zona y convertirse en un propulsor de las iniciativas de mejoramiento ovino, diversificación de cultivos y forestación intensiva.

Por último, planteaban que se debía apuntar a cubrir los requerimientos de toda el área mediante la creación de una "red de escuelas primarias distribuidas en toda la zona teniendo como escuela nuclear o piloto la de Zumbagua, y un Centro Técnico de nivel medio, con especializaciones agropecuarias y artesanales" (Comunidad Salesiana del Ecuador s. f., 20). Este punto es relevante, pues evidencia que la iniciativa de crear un sistema de escuelas en la zona no fue una decisión de última hora o que se dio al azar, sino que estuvo contemplada desde un inicio.

Si bien es cierto que la educación fue un ámbito tematizado por la Comunidad Salesiana desde su llegada a Zumbahua en 1971, también es cierto que la educación fue un tema de preocupación recurrente de los líderes de las comunidades indígenas. Por razones que luego desarrollaremos con detenimiento, los líderes indígenas vieron la necesidad de que la población de la zona se alfabetizara y educara, demanda que fue planteada de manera clara y contundente en el Mandato de Cachi.

En los inicios de los 70 se reunieron en la comunidad de Cachi Alto, parroquia de Pujilí, cerca de un centenar de líderes indígenas de esta y otras provincias con el objetivo de tratar el tema de la organización de la población campesina e indígena de Cotopaxi. Los indígenas 
invitados fueron seleccionados de tal manera que aportaran con el relato de las experiencias organizativas de sus respectivas provincias: este fue el caso, por ejemplo, de los líderes de Simiatug, parroquia del cantón Guaranda, en el que se había desarrollado un proceso organizativo consistente (Toaquiza 1993).

En dicha reunión nació la organización que hoy se conoce con el nombre de MICC, y se llegaron a los primeros acuerdos en torno a su proyecto político, a los que se les dio el nombre de Mandato de Cachi. De acuerdo con el padre José Manangón, ${ }^{14}$ sacerdote que trabajó en la zona desde los 70 y que ha dirigido el proyecto educativo desde finales de la década de los 80, las cinco demandas centrales del mandato fueron las siguientes: tierra propia, organización propia, educación propia, comunicación propia e iglesia propia; demandas que dan cuenta del sentido y alcance del proyecto político que estaba fraguándose en las zonas rurales de la provincia de Cotopaxi. Conociendo la experiencia educativa de los salesianos, los líderes de las comunidades indígenas decidieron delegar al equipo de la Comunidad Salesiana presente en Zumbahua la estructuración del proyecto educativo de la zona.

Por lo anteriormente dicho, vemos que la creación de las escuelas indígenas fue el resultado de la confluencia del proyecto de los salesianos y las expectativas de líderes indígenas de la zona, y que fue justo la imbricación de ambos proyectos e intereses lo que dio la fuerza y la sostenibilidad necesarias a la iniciativa. ${ }^{15}$

14 Padre José Manangón y Mery Martínez, entrevista personal, 10 de abril de 2014, Quito.

15 El protagonismo de la iniciativa educativa es un tema polémico, pues mientras algunos actores como el padre Javier Herrán, sacerdote salesiano que inició el proyecto de las escuelas en la zona, considera que fueron los salesianos los mentalizadores y protagonistas de dicha iniciativa, otros, como el padre José Manangón y varios de los educadores comunitarios, sostienen que fueron los líderes indígenas de la zona. En el fondo, una y otra perspectiva buscan justificar lo actuado por unos y otros, luego de una fuerte crisis que se vivió al interior del equipo salesiano que trabajó en la zona. Nuestro punto de vista es el que acabamos de presentar: la iniciativa educativa en cuestión fue el resultado de la confluencia de intereses de los líderes indígenas y del equipo misionero. 
Móviles que impulsaron la creación de las escuelas indígenas

¿Cuáles fueron los móviles que impulsaron la creación de las escuelas propias en la zona? De entrada, podemos decir que el móvil más inmediato fue el analfabetismo. Para 1974, dos años antes de la creación de la primera escuela indígena, el analfabetismo afectaba a más del $80 \%$ de la población mayor de ocho años de la zona en cuestión. Rodrigo Martínez y Bolívar Burbano, personas que participaron en el proyecto de las escuelas indígenas y que sistematizaron la primera etapa de la experiencia, se refieren a la problemática de la siguiente manera:

En el año de 1974, en un sondeo hecho por el Equipo Pastoral de Zumbahua (EPZ) para conocer el índice de analfabetismo en las zonas de Chugchilán, Zumbahua y Guangaje, se detectó que había una población de 34024 (Chugchilán: 7686; Zumbahua: 14 818; y Guangaje: 11 520), de los cuales 27193 (Chugchilán: 6149; Zumbahua 11 855; y Guangaje: 9 189) era mayores de ocho años de edad. El índice de analfabetismo en la zona de Chugchilán ascendía al 80,7 \%; en la zona de Zumbahua, al 87,3 \%; y, en la Guangaje, al 90,2 \%. A nivel del cantón de Pujilí el índice de analfabetismo era del 54,8 $\%$ y a nivel provincial el 45,4 \%. (Martínez y Burbano 1994, 126)

El analfabetismo en la zona se explica por varias razones, siendo las dos principales: la ausencia del servicio educativo y su baja calidad en aquellos lugares donde sí había escuelas. Con relación al primer tema, en varias de las comunidades indígenas de la zona no existían escuelas, y, las más cercanas, que por lo general se ubicaban en las cabeceras parroquiales o al filo de la carretera, eran inaccesibles para los niños y las niñas por la distancia y el tiempo que utilizaban para llegar (Martínez y Burbano 1994). No es azaroso, entonces, que varias de las primeras escuelas indígenas fueran creadas justo en las comunidades más alejadas, con mayores problemas de acceso, y en las que, por obvias razones, no existían.

Sobre el segundo tema, el relacionado con la calidad, las pocas escuelas públicas que operaban en la zona tenían serios problemas y limitaciones de todo tipo, y no aseguraban las condiciones básicas para el aprendizaje y menos aún un trato respetuoso a los niños indígenas. Martínez y Burbano se refieren a dichas escuelas de la siguiente manera: 
Aquellas escasas escuelas tenían graves deficiencias como: infraestructura que atentaba a la dignidad humana, carencia de recursos didácticos, profesores formados para mantener relaciones de discriminación y dominación, programas de enseñanza pensados desde los sectores urbanos. Por otra parte, los niños eran objeto de inferiorización por parte de los profesores "golondrinas" que desconocían completamente el idioma y desvalorizaban la cultura indígena. Todas estas situaciones describen la crítica situación de las escuelas rurales en las comunidades indígenas de la provincia de Cotopaxi. $(1994,41)$

Las deficiencias señaladas, más la dinámica de la vida indígena de la zona, explican uno de los problemas educativos recurrentes en aquella época, a saber, la temprana deserción de la población indígena del proceso de escolarización: en el tercer grado se retiraba aproximadamente el $60 \%$ de los estudiantes, y los que lograban continuar el proceso lo hacían con mucha irregularidad en materia de asistencia y concluían con dificultad la formación primaria (Martínez y Burbano 1994).

La situación que acabamos de presentar no es muy distinta a la que se vivía en otras zonas rurales de Ecuador, y, en especial, en aquellas en las que se impulsaron iniciativas de educación propia. Si se analiza, por ejemplo, el contexto en el que nacieron las escuelas autogestionarias de Simiatug, provincia de Bolívar, o las escuelas clandestinas de Cayambe, con Dolores Cacuango a la cabeza, se puede constatar que los niveles de analfabetismo, deserción, repitencia y bajo nivel de adecuación de las propuestas educativas eran iguales o peores que los reportados en Zumbahua y Chugchilán. ${ }^{16}$

Ahora bien, ¿por qué el analfabetismo se convirtió en un problema en Zumbahua, en la década de los 70? La pregunta es relevante puesto que se trata de una zona en la que siempre hubo altos niveles de analfabetismo, que fue una problemática tratada por los líderes indígenas debido a que lo veían como un problema para el trabajo, impulsaban las

16 Para el caso de las escuelas autogestionarias de Simiatug se puede revisar el texto de Francesco Chiodi: La educación indígena en América Latina. México, Guatemala, Ecuador, Perú y Bolivia (1990). Para el caso de las escuelas clandestinas de Cayambe se puede revisar el texto de Raquel Rodas: Crónica de un sueño: Las escuelas indígenas de Dolores Cacuango (1989). 
comunidades para recuperar la tierra y controlar los procesos de comercialización de sus productos.

Acerca del primer punto, el analfabetismo constituía una traba tenaz en los procesos de negociación de las tierras, pues los indígenas eran frecuentemente engañados por los dueños de las haciendas y sus abogados. Joselino Ante, uno de los dirigentes que lideró la lucha por la tierra en Guayama y que impulsó la creación las escuelas indígenas en la zona, expresa muy claramente esta situación:

En aquellos días, los líderes, que éramos fuertes y todo, pero no podíamos entender el español, menos leer, entonces éramos engañados, luego el licenciado de Quito nos dijo: "pero ya firmaron, ¿ora qué?" Y eso estuvo lleno de problemas. Entonces luego pensamos, necesitamos aprender a leer, para que estos no nos vuelvan a engañar. Entonces de ahí muchos pensamos que necesitamos una educación indígena, una educación para los indígenas. (Lazos y Lenz 2004, 13)

Aspecto que es corroborado por el padre José Manangón. Al preguntarle sobre el inicio de las escuelas indígenas, contestó lo siguiente:

Hay dos o tres cosas que confluyeron ahí. La primera es que se inició la lucha por la tierra. Entonces se ganaban los juicios, pero cuando hacían las escrituras le devolvían (las tierras) al dueño. O sea, los otros, los comuneros, contentos con las escrituras diciendo "vean taiticos esta es la escritura, es de ustedes", iban a la comunidad y hacían ahí leer y resultaba que quedaba de dueño el mismo hacendado. Entonces, ese fue un momento en que decidieron jya basta! Yo recuerdo haber estado en este momento con los de Guayama, que le ganan la hacienda al patrón, y cuando el patrón les entrega las escrituras, llegan a Guayama a hacer la fiesta, y antes de prender los voladores y la fiesta hacen leer la escritura y resulta que el dueño seguía siendo el hacendado. Entonces, ahí decidieron ¡ya punto! Aquí hay que definitivamente aprender a leer y escribir. ${ }^{17}$

Con relación al segundo punto, el tema de la comercialización, tenemos que, junto con la lectura y la escritura del español, el cálculo fue otro tema de preocupación para la población indígena, pues su desconocimiento constituía un serio problema en el momento de pesar los

17 Padre José Manangón y Mery Martínez, entrevista personal, 10 de abril de 2014, Quito. 
productos y realizar las cuentas con la población mestiza, eventos en los que, al igual que en el caso de las negociaciones por la tierra, los comuneros salían perdiendo.

El padre Manangón comenta lo siguiente sobre el tema: "En Zumbahua era, en cambio, el tema del comercio. Los negociantes de Saquisilí les estafaban en cuanto al peso de los productos. Les compraban los quintales de cebada con ciento cincuenta libras, ciento sesenta libras. Era impresionante". ${ }^{18}$ En la misma línea, Lazos y Lenz expresan lo siguiente: "Aunque no fueran grandes las cantidades de productos que se comercializaban, el abuso por parte de los blancos denigraba constantemente a los indígenas. Esto llevó a que los indígenas lucharan por conocer los números y saber las cuentas para poder defenderse" $(2004,14)$.

Existe una tercera razón que, según el padre Manangón, hizo que la escritura fuera vista por los líderes indígenas como una herramienta clave en la vida de las comunidades. Conforme pasaba el tiempo, la población de la zona tenía más vínculos y relación con las instancias locales del Estado y con la sociedad blanco-mestiza en general, y en dichas relaciones la firma se convirtió en un elemento totalmente necesario: tenían que firmar para tramitar créditos en el Fondo de Desarrollo Rural Marginal (FODERUMA), para solicitar una carretera en la prefectura, para realizar cualquier trámite en el municipio; otra necesidad que llevó a que la población indígena se interesara por el aprendizaje de la lectura y escritura.

Si bien es cierto que el móvil inmediato para la creación de las escuelas indígenas fue la necesidad de erradicar el analfabetismo que campeaba en las comunidades, hubo incluso otras motivaciones que, aunque no tenían una relación directa con el tema educativo, no por ello eran menos importantes para estas. De acuerdo con Martínez y Burbano (1994), una de las motivaciones para la creación de las escuelas era la necesidad de las comunidades de buscar reconocimiento frente a las otras, pues para la población indígena contar con una escuela —además de una casa comunal, una capilla y un cementerio- era síntoma de que la comunidad estaba saludable y saliendo adelante. Era su motivación más frecuente. 
La segunda motivación fue la necesidad de fortalecer el proceso organizativo para la lucha por la tierra. La escuela fue vista, en varias comunidades indígenas, como una instancia clave para aglutinar a la población y organizarse para esa lucha, que constituía el tema del día a día en Guayama, Pilapuchín, Chinaló, entre otras.

Una tercera motivación tenía que ver con la necesidad de neutralizar el autoritarismo de una o varias familias poderosas al interior de las mismas comunidades. En este caso, la escuela fue concebida como un poder paralelo y una instancia desde la cual hacer frente a dichas familias. Este fue el principal móvil en Zumbahua y Guangaje, zonas en las que, luego del proceso de repartición de las tierras de las antiguas haciendas, ciertas familias indígenas comenzaron a reproducir la misma dinámica del poder de los hacendados.

Una última motivación que merece ser señalada es aquella que se refiere a las expectativas que las comunidades tenían sobre los docentes y en especial sobre sus actitudes. Varias escuelas indígenas fueron creadas con la finalidad de liberarse de "la irresponsabilidad y el maltrato del que fueron objeto por parte de los profesores mestizos hispanohablantes" (Martínez y Burbano 1994, 148). La escuela indígena, en estos casos, fue asumida como la posibilidad de contar con un docente indígena de la misma comunidad, comprometido con la educación de niños y niñas y el desarrollo de la comunidad.

Lo anteriormente dicho nos permite entender un hecho a veces bastante incomprendido: aquel que hace alusión al carácter multifuncional de la escuela indígena. ${ }^{19} \mathrm{Y}$ es que, desde un inicio, la escuela propia fue asumida por las comunidades indígenas como una instancia cuyas funciones no se agotaban en el proceso de enseñanza de ciertas competencias sentidas como necesarias, sino como una instancia central en la

19 José Sánchez Parga (2005) cuestiona fuertemente la iniciativa educativa en cuestión debido a que sobrevaloró la dimensión política y descuidó la pedagógica-educativa. De allí, inclusive, el nombre de su libro: Educación indígena en Cotopaxi: Avances políticos y deudas pedagógicas. Desde nuestro punto de vista, el problema es que Sánchez Parga analiza la iniciativa educativa partiendo de una premisa que no es real, a saber: que el papel central de las escuelas indígenas fue la enseñanza-aprendizaje de ciertas competencias en los estudiantes. 
vida de la comunidad, alrededor de la cual se organizaba la comunidad y se impulsaban acciones colectivas orientadas al mejoramiento de las condiciones de vida de los comuneros.

Es en este marco que deben ser entendidas las afirmaciones señaladas (afirmaciones repetidas hasta el cansancio tanto por los educadores comunitarios como por los líderes indígenas) de que la principal motivación para crear las escuelas fue, por ejemplo, la lucha por la tierra o la neutralización del poder de los cabecillas. En dichas afirmaciones está implícita la concepción de la escuela como una instancia organizativa y de promoción de la comunidad, con fines netamente políticos, antes que como un espacio de enseñanza-aprendizaje. ${ }^{20}$

\section{Nacen las escuelas indigenas}

Con base en las motivaciones anotadas, los dirigentes indígenas y el equipo de la Pastoral de Zumbahua apostaron por la creación de un servicio educativo en la zona. En un inicio los esfuerzos se orientaron a crear centros de alfabetización en los que participó, fundamentalmente, la población adulta de las comunidades. En un segundo momento, con la experiencia acumulada y por pedido de las comunidades, los esfuerzos se volcaron a la creación de las escuelas indígenas.

En este punto vale aclarar que, años antes de la creación de las escuelas indígenas, el equipo de la Pastoral de Zumbahua orientó sus esfuerzos a gestionar, ante el Estado, escuelas públicas para las comunidades, pero luego de una experiencia totalmente negativa en las comunidades de Guasumbiní y Chinaló, en las que se pudo constatar que lo único que se había logrado era crear escuelas fiscales con los mismos problemas y limitaciones ya señaladas, se decidió apostar por la creación de escuelas propias, al margen del Estado (Íñiguez y Guerrero 1986).

En 1976 se creó la primera escuela indígena en la comunidad de Pilapuchín, parroquia de Chugchilán, que comenzó a funcionar en la casa del presidente de la comunidad de ese momento, y con un voluntario de Quito como docente. El padre Javier Herrán, sacerdote salesiano que jugó

20 Esta dimensión de la escuela indígena la profundizaremos en el siguiente apartado. 
un papel central en la creación de las primeras escuelas indígenas, recuerda de la siguiente manera lo acontecido durante los primeros años:

Recordar el inicio de las Escuelas Indígenas es hablar de Pilapuchín, de Paco Velasco y de Lorenzo Licta, entonces presidente de la comunidad de Pilapuchín. La casa de Lorenzo Licta comenzó siendo escuela, dormitorio y cocina, donde Paco sacaba punta a los primeros lápices que conocían en Pilapuchín. Las pocas palabras kichwas que Paco aprendió sirvieron para comenzar el proceso de lecto-escritura de jóvenes y niños. Era el mes de octubre del año 1976.

Para el año siguiente, la escuela se había convertido en algo necesario para la comunidad de Pilapuchín. La capilla de la comunidad abrió sus puertas para que se aprendiera a "pensar, leer y contar", a la vez que se rezara a Dios y se luchara por los derechos al agua y al pasto. Feliciano Sigcha de la comunidad de Itualó se comprometió a continuar con el trabajo de Paco.

En el año de 1978 ya no era solo Pilapuchín, sino también Chaupi, Guayama-Cuisana, Quindigüa y Sarahuasi. A Feliciano se sumaron Manuel Guamangate, Benedicto Tigasi, Francisco y Pedro Cunuhay; todos de la comunidad de Itualó — que en ese tiempo era como la Universidad del Páramo- - Esa era la única comunidad donde muchos jóvenes habían acabado la primaria. $(2008,53)$

Como se puede constatar en el testimonio del padre Javier Herrán, en un inicio las escuelas indígenas operaron en espacios totalmente improvisados, como la casa del dirigente de la comunidad, una choza cedida por los comuneros o la capilla de la comunidad, en caso de que hubiera; sin el mobiliario necesario y menos aún con el material didáctico requerido. Con el pasar de los años, las comunidades indígenas, con el apoyo del equipo de la Pastoral y el aporte financiero de ciertas instituciones (FODERUMA, por ejemplo), lograron construir al menos un aula y amoblarla con lo básico para el trabajo educativo.

En la misma línea se puede constatar también que, en las primeras escuelas indígenas, los educadores no fueron indígenas de las comunidades en las que se creaban las escuelas o de comunidades aledañas, sino voluntarios que se encontraban apoyando al equipo de la Pastoral en el desarrollo de su proyecto misionero, lo que es totalmente entendible 
si se considera que en la zona eran muy pocos los comuneros que habían concluido la educación primaria y menos aún los que tenían algún conocimiento y experiencia pedagógicos. Al poco tiempo, sin embargo, aquellos comuneros que habían logrado concluir la educación primaria y que habían tenido una trayectoria importante en la comunidad reemplazaron a los voluntarios y se hicieron cargo de las escuelas. ${ }^{21}$

Conforme se fueron consolidando las escuelas de las comunidades señaladas por el padre Javier Herrán, y conforme se fue expandiendo la noticia en la zona, otras comunidades comenzaron a demandar y gestionar sus propias escuelas, $y$, de manera paulatina, la experiencia educativa se fue ampliando hacia otras parroquias y cantones. En el transcurso de la década de los 80 los centros educativos se multiplicaron y, para 1988, año en el que el equipo de apoyo y educadores de las escuelas decidieron realizar el primer ejercicio de sistematización y evaluación de la experiencia con el objetivo de lograr el reconocimiento del Estado, se encontraba consolidada una red educativa que la conformaban veintiún escuelas indígenas, quince huahuacunapac-huasi (guarderías) y diecinueve centros de alfabetización; que cubría la demanda educativa de las comunidades indígenas de las parroquias de Chugchilán, Zumbahua, Guangaje, El Corazón, Pujilí y Poaló, estas dos últimas conocidas como la zona de El Bajío (SEIC 1989).

Como era de esperar el proceso de creación de las escuelas indígenas no resultó fácil: fue un trabajo que generó mucha resistencia por parte de los hacendados y otros actores externos a la comunidad (comerciantes, chulqueros y autoridades), pero incluso por parte de los mismos comuneros.

Desde el punto de vista de los hacendados y demás actores externos, la escuela y el proceso de educación de los indígenas era visto como un riesgo para el orden y estabilidad de la zona, lo que les llevó a poner en juego todas las estrategias posibles para evitar que siguieran naciendo o se consolidaran. En varias ocasiones los hacendados reaccionaron con violencia, como fue el caso, por ejemplo, de lo que ocurrió en la

21 En el siguiente acápite hablaremos sobre los educadores comunitarios y el perfil solicitado por las comunidades para hacerse cargo de las escuelas indígenas. 
comunidad de Guayama. Joselino Ante, dirigente de dicha comunidad, comenta lo siguiente sobre los primeros momentos de la escuela, cuando aquella estaba a cargo de una profesora voluntaria italiana:

La compañera Emiliana sufrió mucho por ofrecerse a darnos sus clases. Por la noche, los patrones iban a ponerle candela en la casa que ella dormía. No sé cómo se sentiría cuando ellos prendían candela; sé que se levantó y gritó, entonces, nosotros fuimos a defenderla. Sé que hubo bastantes problemas para conseguir la educación, pero los niños empezaron aprendiendo a leer y escribir, porque nadie sabía. (Díaz 1997, 15)

Lo anterior explica el porqué del carácter clandestino de las escuelas, sobre todo de las que nacieron en comunidades en las que se vivía mucha agitación política por el tema de la lucha por la recuperación de la tierra. Y es que la clandestinidad constituía, en ese momento, una estrategia clave para la sobrevivencia de la escuela. Lo anterior nos recuerda la historia de las escuelas indígenas impulsadas por Dolores Cacuango en Cayambe, que, en los primeros momentos, también operaron en la clandestinidad debido a la reacción de los hacendados de la zona en contra de los líderes indígenas y los educadores (Rodas 1989).

La resistencia no solo venía desde el lado de los hacendados, comerciantes y demás actores externos a la comunidad, sino incluso, y muchas veces, de los mismos comuneros, en especial de aquellos que habían plegado a los hacendados y se habían apropiado de su discurso (SEIC 1989).

Fue arduo el trabajo que tanto los líderes indígenas como el equipo de la Pastoral desplegaron para convencer a los comuneros sobre la necesidad de la escuela propia. Las estrategias utilizadas para el convencimiento fueron de diferente índole, siendo el chantaje una de las posibles. A manera de ejemplo, revisemos lo que Joselino Ante dice sobre la estrategia que utilizaron para asegurar que los padres de familia de la comunidad de Guayama enviaran a sus hijos a la escuela:

La gente no quería hacerle caso (a la profesora italiana), porque pensaba que los gringos iban a comerse a nuestros hijos y se los llevarían para venderlos. Hubo muchas broncas por causa de la educación, tuvimos que portarnos bastante duros con el padre Javier. Él decía que nosotros 
somos hijos de Dios, y si somos personas, debemos ser educados. Les decía a los compañeros "si ustedes no quieren dar educación, entonces sus hijos tampoco son hijos de Dios. ¡Qué! ¿son hijos de animales?”.

En último caso, le recordé al padre Javier Herrán que no era conveniente bautizar porque ellos no parecían cristianos, parecían hijos de animales, que no eran hijos de cristianos. En ese caso, el padre Javier dijo "no bautizaré ni casaré". Con ese miedo empezaron poniéndoles en la escuela; iniciaron catorce alumnos a inicios de noviembre de 1976. (Díaz 1997, 14-5)

La alusión a que los "gringos iban a comerse a nuestros hijos y se los llevarían para venderlos" es interesante por cuanto da cuenta de las representaciones (Hall 2010) construidas por los hacendados, autoridades de la zona y comerciantes para deslegitimar el trabajo de los misioneros y los líderes indígenas, en general, y en materia educativa, en particular. La primera era la etiqueta de "gringos" para referirse a los misioneros salesianos y de Mato Grosso, buena parte de los cuales eran italianos y españoles, y cuyos rasgos fenotípicos marcaban una diferencia grande con los de la población indígena de la zona. La otra etiqueta era la de "comunistas", también para referirse a los misioneros, pero asimismo a su equipo de apoyo y a los líderes indígenas. Ambos membretes estaban cargados de connotaciones altamente negativas: ladrones, traicioneros y peligrosos, entre otras.

La falta de apoyo por parte de los padres de familia se debía no solo a la influencia de los hacendados, pues muchas veces tenía que ver también con la concepción que los padres tenían de los niños y las niñas, así como con el hecho de que aquellos no creían en la propuesta educativa en ciernes ni en la capacidad de los educadores comunitarios. Respecto al primer punto, era común, en los primeros años de la experiencia, que los comuneros enviaran a la escuela únicamente a sus hijos varones, pues consideraban que sus hijas tenían que quedarse en la casa apoyando en las labores domésticas y aprendiendo el oficio. Carmen Martínez hace referencia a esta situación y a la manera en que el padre Javier Herrán solía enfrentarla:

Viendo que acudían muy pocas niñas a la escuela [el padre Javier Herrán], pidió a los campesinos que mandaran a sus hijas. Ellos respondieron que las mujeres no necesitaban estudiar, nomás para cuidar la 
casa, los niños y pastar borregos. El padre dijo: “¿Ustedes creen que las mujeres no son seres humanos como los hombres? Si eso es lo que ustedes piensan, entonces a partir de ahora no bautizaré a las niñas". Los campesinos aterrados por este prospecto decidieron mandar a las niñas a la escuela. $(2004,261)$

Con relación al segundo punto, la resistencia de los comuneros a las escuelas indígenas tenía que ver incluso con la imagen devaluada que tenían de ellas (la escuela fiscal era el referente máximo) y así como de las capacidades de los educadores comunitarios que no tenían título. Leonardo Cunuhay, educador comunitario de la escuela de la comunidad de Moreta, explica esta problemática en los siguientes términos:

Los primeros años de trabajo de este tipo de educación algunos compañeros comuneros no entendían el significado de tener la educación bilingüe en la comuna y trataban de poner trabas a la educación ya que todos tenían centrada la ideología de la educación fiscal, pues nuestra educación es diferente a la educación por medio de metodología, pedagogía.

Los problemas que aparecían es por los comentarios en contra de la educación bilingüe incluso cuando se emborrachaban venían a reclamar por nuestra presencia en la comunidad, como en ese entonces los compañeros educadores no tenía el título pero aún los criticaban diciendo que ellos no saben, eran tiempos difíciles, pero los educadores fueron haciendo conciencia y entender el significado de nuestra educación. $\mathrm{Al}$ principio algunos padres de familia estaban en contra de la educación bilingüe, por lo que no mandaban a sus hijos a la escuela, en estos momentos toda la comunidad está con entusiasmo de llevar adelante la educación, pero existe aún gente como la de Chugchilán que trata de separar a los comuneros con cuentos pero ellos más bien discuten sobre el asunto y ya no se dejan engañar. $(1993,16-7)$

Lo anterior es interesante no solo porque demuestra la falta de convencimiento que tenían, al inicio, varios comuneros sobre las escuelas indígenas, sino porque da una idea de las múltiples actividades que los educadores comunitarios debían realizar pues, a la par del proceso de enseñanza, debían preocuparse por llevar adelante todo un trabajo de concientización con los padres de familia para contrarrestar el trabajo de manipulación de los hacendados y demás actores empeñados 
en bloquear el funcionamiento de las escuelas. En el siguiente apartado profundizaremos sobre el rol del educador comunitario.

La resistencia por parte de los comuneros hacia las escuelas indígenas se demostraba por medio de críticas permanentes hacia estas y los educadores, pero, sobre todo, mediante la negativa de inscribir y enviar a sus hijos. En muchos casos, dicha resistencia terminó poniendo en crisis a las escuelas y, en otros, los menos, cerrándolas. El siguiente testimonio de Ernesto Baltazaca, primer educador de la escuela de la comunidad de Saraucsha, da una idea de la magnitud del problema:

En todas las comunas había analfabetos y entonces en las reuniones comenzamos a hablar de la necesidad de la educación. Tanto estar conversando, llegó el día de ir a un cursito en Cachi Alto; cuando regresé de ahí [noviembre de 1977] comencé el trabajo en mi comunidad. Al principio había sesenta alumnos, pero la gente murmuraba, insultaba, diciendo ¿cómo va a poder enseñar, uno que no es estudiado, que no es graduado? Tanto que chismeaban, se fueron muchos alumnos, solo quedaron seis $\mathrm{u}$ ocho, ellos sacaron el certificado de haber terminado la primaria. Viendo esto, los otros se animaron nuevamente a seguir. (SEIC 1989, 88)

Para cerrar este apartado es necesario decir que, luego de doce años de vida y de un largo trabajo de negociación con el Estado, las escuelas indígenas del Quilotoa fueron finalmente reconocidas por este en 1988, y pasaron a depender del Departamento de Educación Compensatoria y no Escolarizada de la Dirección de Educación de Cotopaxi, hasta unos meses después que pasó a depender de la naciente DINEIB (Ante 1993).

Las razones que llevaron al equipo de apoyo y a los líderes comunitarios a buscar la oficialización de la experiencia, y los cambios que el proceso de reconocimiento por parte del Estado trajo para las escuelas indígenas, son temas de discusión en el tercer capítulo. Por el momento, pasamos a analizar la propuesta educativa y el modelo de gestión que las escuelas indígenas pusieron en marcha para atender las demandas de formación de las comunidades indígenas. 


\section{El proyecto educativo y el modelo de gestión}

Para los salesianos constituyó una gran responsabilidad y reto que los líderes indígenas les encomendaran la estructuración del proyecto educativo para formar a la población indígena de la zona, que era numerosa, con un nivel de analfabetismo muy alto, y en condiciones económicas, políticas y sociales para nada favorables.

$\mathrm{Al}$ interior del equipo misionero se generó un intenso debate en torno al tipo de educación que se debía implementar en la zona. Había claridad en cuanto a que la propuesta educativa debía, necesariamente, desmarcarse de lo que hasta ese momento constituían las dos propuestas educativas más cercanas para los salesianos: la educación privada, por un lado, y la educación fiscal, por el otro. El problema con la educación privada era su carácter elitista y su orientación a los requerimientos del modelo económico dominante, mientras que el problema con la educación fiscal era su baja calidad. El padre José Manangón explica la tensión vivida:

Yo recuerdo un debate muy intenso de cómo debía ser la educación definitivamente fuera del Estado. Decíamos "no podemos caer en los mismos problemas de la educación del Estado, tampoco podemos caer en los mismos problemas de la educación salesiana". Y el ejemplo que poníamos siempre era el [colegio] Spellman, el Santo Tomás, el Técnico de Cuenca [...]. Veíamos dos cosas negativas en esas experiencias, lo elitista, en primer lugar, y eso estaba alineado, definitivamente, al sistema capitalista. Entonces no se podía repetir ese asunto. Y también debíamos guardar cierta distancia con la educación del Estado, porque era pésima. Entonces, no se podía caer ahí, en la educación que proponía la UNE, el MPD, eso no. Decíamos: "hay que hacer una cosa propia que nazca desde la cultura y desde la realidad", y evidentemente eso estaba sustentado en lo de Paulo Freire. ${ }^{22}$

Con el tiempo, el equipo misionero y los líderes indígenas ganaron claridad y lograron acuerdos sobre el carácter y alcance de la propuesta educativa. Entre los temas de consenso se encontraba aquel relacionado con la necesidad de generar una propuesta que naciera de la realidad de la zona y se adecuara a ella, ${ }^{23}$ lo que es totalmente entendible

22 Padre José Manangón y Mery Martínez, entrevista personal, 11 de abril, Quito.

23 Ibíd. 
si se considera, como vimos arriba, los graves problemas y limitaciones de la educación pública que se implementaba en dicha época.

Por una educación "que naciera de la realidad y se adecuara a ella" se entendían varias cosas: un proyecto educativo que se organizara con base en los requerimientos y necesidades de las comunidades indígenas de la zona, que se alineara con sus causas y proyectos de corto, mediano y largo plazos; que considerara y acoplara a sus tiempos y dinámica de vida; que utilizara la lengua materna como el medio fundamental de comunicación y aprendizaje; que fuera impartida por personas de las comunidades, y que fuera regentada y orientada por las mismas comunidades. A este tipo de educación es a la que los líderes indígenas y el equipo misionero denominaron educación propia.

Ahora bien, ¿se puede sostener que la educación implementada por las escuelas indígenas fue una iniciativa que nació y se adecuó a la realidad de la zona? Luego de revisar con detenimiento la documentación generada sobre las escuelas (estudios y evaluaciones) y conversar con los gestores y docentes que acompañaron a las escuelas en los primeros doce años de vida, podemos decir que el proyecto educativo implementado por las escuelas indígenas fue, sin lugar a dudas, uno que se organizó sobre la base de los requerimientos de la zona y se adecuó al contexto y dinámica de vida de su población. Pasemos a analizar algunas aristas del proyecto educativo, para luego centrar la atención en el modelo de gestión con el que operaron las escuelas.

El proyecto educativo de las escuelas indígenas

Se trató de un proyecto educativo que apuntó a responder a los requerimientos del momento por medio de la puesta en juego de un plan de estudios y una metodología idóneas para ello. Revisemos cada uno de estos elementos.

Una de las metas del proyecto educativo impulsado por las escuelas fue desarrollar en los estudiantes indígenas las habilidades necesarias para enfrentar las situaciones problemáticas que tenían que vivir en el día a día, en su relación con los diferentes actores de la población mestiza de la zona. Aquellas habilidades eran la lectura, la escritura, y 
el manejo de las cuatro operaciones matemáticas básicas: suma, resta, multiplicación y división. ${ }^{24}$

Como adelantamos, dichas habilidades fueron concebidas por los comuneros como fundamentales para defenderse de los hacendados y abogados en los procesos de negociación de las tierras, de los comerciantes y chulqueros en los procesos de compraventa y solicitud de préstamos, de las autoridades locales en los diferentes trámites que debían realizar, y, en general, en la vida cotidiana fuera de sus comunidades: los comuneros que viajaban a Quito u otras ciudades, ya sea para realizar trámites de diferente índole o buscar recursos económicos alternos, veían como un verdadero problema, por ejemplo, no poder leer las rutas de los buses, pues continuamente se perdían en la vía o al interior de las ciudades. Además, como ya dijimos, estaba el tema de la "firma", aspecto de mucha importancia en aquella época en la que la población indígena tenía cada vez más contacto con el mundo no indígena.

La segunda meta, y que estaba íntimamente conectada con la anteriormente descrita, era aquella que tenía que ver con el desarrollo de un pensamiento propio en los estudiantes. Esto, al igual que el propósito anotado, era de una importancia capital para los líderes indígenas y el equipo de apoyo, pues se consideraba que uno de los factores que explicaba la situación deplorable en la que vivía la población indígena era el hecho que no había tenido "capacidad para pensar por su propia cuenta". 25

El desarrollo de un pensamiento propio ("pensar con cabeza propia" era el lema del momento) implicaba, entre otros aspectos, la capacidad para analizar, desde su condición de indígenas y desde una perspectiva crítica, su historia y situación del momento, de reconocer y valorar su condición cultural y potencialidad como pueblo, de proyectarse al futuro en términos colectivos, entre otros; elementos centrales para su proyecto de reconstrucción como pueblo, en todas sus dimensiones. ${ }^{26}$

24 Padre Javier Herrán, entrevista personal, 14 de julio de 2014, Quito.

25 Padre José Manangón y Mery Martínez, entrevista personal, 11 de abril de 2014, Quito.

26 Ibíd. 
Para el trabajo en el día a día el proyecto educativo se organizó en tres áreas formativas que estaban interconectadas entre sí: Académica, Formación humana y Actividades prácticas. La Académica estaba orientada al desarrollo de las habilidades de lectura, escritura y cálculo, pero también de un conjunto de conocimientos básicos sobre el mundo natural y social; y estaba conformada, a su vez, por cuatro subáreas: idiomas, matemáticas, ciencias sociales y ciencias naturales (Manangón, Baltazar y Trávez 1992).

Con relación a la subárea de idiomas (que incluía kichwa y español), la consigna del momento era que los estudiantes aprendieran a leer y escribir en kichwa pero asimismo en español, por su importancia para las relaciones con la población mestiza, a las que ya nos referimos; para lo cual, los dos o tres primeros años (el tiempo exacto dependía del ritmo de aprendizaje del estudiante, como veremos más adelante) se destinaban a la enseñanza del kichwa para después pasar, de manera gradual, a enfatizar en la enseñanza del español.

En el caso de la subárea de matemáticas, los contenidos eran las cuatro operaciones básicas. Los contenidos de la subárea de ciencias naturales eran: nuestra huerta, nuestro cuerpo y nuestro mundo; y los de la subárea de ciencias sociales: época aborigen, época republicana, la situación actual y nuestra tierra. En el caso del componente histórico de la subárea de ciencias sociales, el reto era doble pues la idea no era solo trabajar los principales momentos de la historia de Ecuador, sino hacerlo desde la perspectiva indígena y visibilizando aquellos hechos y acontecimientos que habían provocado alteraciones importantes en la vida social de la población indígena (Îniguez y Guerrero 1986).

El área de formación humana, por su parte, tenía como propósito central provocar en los estudiantes un proceso de reflexión permanente sobre aspectos clave de su situación como personas pero también como colectividad: su historia, problemas, posibilidades, valores de la convivencia comunitaria, etc. El tema de los valores constituía un tema prioritario de discusión y reflexión en esta área, pues como sostiene el padre José Manangón, ${ }^{27}$ en aquella época existían serios problemas de

\section{Ibíd.}


violencia al interior de las comunidades, que no permitían que estas se cohesionaran y terminaran de organizarse para salir adelante.

Y, por último, estaba el área de actividades prácticas que contemplaba, de manera secuencial, un conjunto de actividades fuera del aula que tenían como finalidad preparar a los estudiantes para el trabajo futuro en la agricultura, la ganadería, el trabajo artesanal, y enseñarles a administrar sus recursos. Entre dichas actividades se encontraban las siguientes: crianza de cuyes, aves y chanchos, preparación y cuidado de huertos, actividades de forestación, artesanía en cuero y sastrería (SEIC 1989).

Con respecto a la metodología de trabajo en el aula, la consigna de las escuelas era partir siempre de la experiencia inmediata de los estudiantes y su práctica, pues el objetivo era que la educación tuviera sentido, pero sobre todo que fuera útil. Esta vinculación, que de por sí era más fácil promover en el trabajo de los contenidos de las subáreas de ciencias naturales y de ciencias sociales, además, se lo incentivaba en el proceso de enseñanza-aprendizaje de los contenidos de la subárea de matemáticas. Revisemos lo que Ruth Moya nos comenta sobre el tema:

La propuesta educativa [de las escuelas indígenas] era genial, porque todo el currículo, óyeme bien, absolutamente todas las áreas curriculares estaban ligadas al proyecto productivo. En otras palabras, el proyecto productivo era la columna vertebral de la propuesta educativa.

Te doy un ejemplo: no me acuerdo el nombre de la comunidad, pero el tema es que en esa comunidad se decidió sembrar lenteja, previo un estudio breve de carácter financiero para ver cómo se la vendería en el mercado. Entonces en la escuela se escogió la lenteja como tema central. Entonces los chicos aprendían cálculo haciendo los surcos, calculando las semillas, calculando el control de plagas, el deshierbe. O sea, en otras palabras, todas las etapas productivas de la lenteja estaban ligadas a la enseñanza de la matemática. Entonces, los chicos hacían cálculos reales, pero en función de la realidad, de la lenteja, en este caso.

Entonces todo el proceso productivo estaba ligado a la enseñanza de la matemática, pero esa era una característica; lo genial era que participaban los chicos de todos los grados y de todas las edades, a quienes se les asignaban tareas de acuerdo a su nivel de manejo de la matemática. Y algo que a mí me sorprendió era la gran capacidad matemática que tenían los chicos, cómo hacían los cálculos en base a una experiencia de la realidad. 
Entonces verás, por ejemplo, cuando ya cosechaban la lenteja, sabían dónde iban a vender, a quién iban a vender, cuánto costaba, cuál era la ganancia, en fin. Entonces, todos los cálculos de la matemática eran en función de la producción de la lenteja. Pesos, medidas, cantidades y como te dije, labores agrícolas a lo largo de la siembra de la lenteja, y también después de la cosecha. Entonces, la matemática estaba ligada a la precosecha y a la poscosecha de la lenteja, en este caso. Pero igual pasaba con la papa o con los otros productos que se producían en la zona. ${ }^{28}$

Para el trabajo en el aula el equipo de apoyo de las escuelas, bajo la orientación del padre Segundo Cabrera, preparó un conjunto de cartillas en las que se plasmaron y desarrollaron los contenidos que se debían trabajar en cada una de las subáreas, así como la secuencia con la que debía hacerse. Los insumos para preparar las cartillas eran de diferente tipo, sin embargo, uno los principales eran los materiales educativos que los mismos educadores comunitarios generaban para su trabajo en el aula: cuentos, dibujos y mapas, entre otros. ${ }^{29}$ Este es uno de los factores que explica por qué las cartillas eran tan valoradas por los educadores y los mismos estudiantes.

Al inicio solo había cartillas para las subáreas de idiomas y matemáticas, debido a las dificultades que el proceso de enseñanza-aprendizaje de los contenidos de aquellas presentaban para los estudiantes, pero con el tiempo se crearon cartillas también para las subáreas de ciencias sociales y ciencias naturales. Para 1986 las escuelas contaban con un total de 36 cartillas para las diferentes subáreas, siendo la mayor parte de ellas para el trabajo de los contenidos de las subáreas de matemáticas e idiomas (Iñiguez y Guerrero 1986).

La estructura de las cartillas variaba dependiendo de la subárea. Las cartillas para el aprendizaje de la historia, por ejemplo, se organizaban por temas ordenados cronológicamente, y cada tema contemplaba una breve exposición de los contenidos centrales, y se cerraba con un conjunto de preguntas que tenían como función evaluar lo aprendido o

28 Ruth Moya, entrevista personal, 7 de abril de 2014, Quito.

29 Fernando Garcés, entrevista personal, 21 de abril de 2015, realizada vía Skype. 
reflexionar, con base en lo aprendido, sobre tópicos relacionados con la vida de las comunidades.

Otras cartillas, aquellas orientadas al cuidado de la salud, por ejemplo, y que pertenecía la subárea de ciencias naturales, se organizaban por temas, pero a diferencia de las cartillas de historia, cada tema se trabajaba con historietas protagonizadas por personajes de la comunidad y basados en situaciones vividas allí. Así, por ejemplo, para hablar de la necesidad de que las madres se cuidaran durante el embarazo se colocaba una historieta sobre una comunera que perdió a su hijo por cargar mucho peso. Se aprovechaba la historieta para, al final, dar orientaciones de cómo cuidarse durante el embarazo y se planteaban preguntas para provocar la reflexión.

Las cartillas de la subárea de idiomas (tanto de kichwa como de español) se estructuraban siguiendo la secuencia del aprendizaje de la lectura y la escritura de las lenguas, que para ese momento estaba fundamentado en el enfoque silábico (Poaquiza 2013) pero, además, bajo el enfoque de la palabra generadora, en la línea de la pedagogía freiriana. ${ }^{30}$

En coherencia con el objetivo de fortalecer la lengua materna, todas las cartillas estaban escritas en kichwa, salvo las que se utilizaban para la enseñanza de la lectura y la escritura del español. En términos didácticos las cartillas seguían varios criterios didácticos, entre los que destacan los siguientes: las cartillas debían partir de la realidad de la zona y preferiblemente de un gráfico, debían ser sencillas e ir de lo simple a lo complejo, y debían estar escritas en letra imprenta para facilitar la comprensión de los estudiantes (Poaquiza 2013).

Vale destacar en este punto también el trabajo visual de las cartillas, producto de la habilidad y pericia del padre Segundo Cabrera. Todas las cartillas incluían ilustraciones que le daban una marca "local", y que creaba sentido de pertenencia y hasta de afecto por parte de los estudiantes y los mismos educadores. ${ }^{31}$

30 Ibíd.

31 Ibíd. 
Desde el punto de vista de los educadores comunitarios, las cartillas constituían el corazón de la propuesta educativa de las escuelas indígenas, pues eran ellas las que realmente comandaban lo que se trabajaba y la manera en que se lo hacía en las escuelas indígenas, además de que constituían el material de trabajo del día a día de los estudiantes. ${ }^{32}$ Las cartillas suplieron, en buena parte, la débil formación con la que contaban los educadores comunitarios en los primeros años de vida de las escuelas indígenas, pues, como veremos más adelante, muchos de ellos, el momento en que comenzaban a trabajar, no habían ni siquiera completado la formación primaria.

Si bien las cartillas fueron el corazón de la propuesta, no fueron los únicos materiales que se utilizaron para el trabajo en el aula. Fueron importantes asimismo los materiales producidos por el Centro de Investigación para la Educación Indígena (CIEI) de la PUCE, ${ }^{33}$ que, pocos años después del nacimiento de las primeras escuelas indígenas, impulsó una experiencia de educación indígena interesante en varias provincias del país, entre las que se encontraba la de Cotopaxi. ${ }^{34}$

En el marco de dicho proyecto se produjeron abundantes textos y manuales en kichwa para el trabajo en todas las áreas del conocimiento, que fueron utilizados por los profesores de las escuelas indígenas, en especial para el trabajo en los primeros niveles. Dos eran las ventajas del material generado por el CIEI: la primera era que discutían con amplitud y profundidad temas que no eran tratados, o que eran tratados de manera superficial, por las cartillas de las escuelas indígenas; y la segunda era

32 Todos los educadores comunitarios se refieren en muy buenos términos a las cartillas. Algunos de ellos, inclusive, las seguían utilizando hasta mediados de 2014, fecha en la que concluyó el trabajo de campo de la presente investigación. Entre las virtudes destacadas de las cartillas es que eran materiales que definían con claridad qué es lo que se tenía que trabajar en el aula, pero también cómo trabajarlo: secuencia y actividades.

33 Padre Javier Herrán, entrevista personal, 14 de julio de 2014, Quito.

34 En el capítulo III haremos referencia al CIEI y a su modelo educativo MACAC, denominado así porque la experiencia educativa piloto fue aplicada en la comunidad de Macac Grande, ubicada en Cotopaxi, cerca de las comunidades en las que operó el SEIC (Yánez 1996). 
que dicho material incluía muchos dibujos y color, lo que gustaba a los estudiantes (Íñiguez y Guerrero 1986).

Un tema innovador de la propuesta educativa de las escuelas indígenas, además de los ya expuestos, es el relacionado con los niveles de aprendizaje. A diferencia de la educación pública del momento, que contemplaba para la educación primaria seis años de estudio, y en la que los estudiantes avanzaban juntos al curso superior, previa aprobación del curso inferior; la propuesta educativa de las Escuelas Indígenas del Quilotoa se organizó con base en niveles que estaban comandados por los contenidos de las cartillas aludidas: un nivel era equivalente al aprendizaje de los contenidos de una o varias cartillas. Aquellos estudiantes que concluían con el aprendizaje de los contenidos de un nivel, inmediatamente, continuaban con el siguiente nivel, sin la necesidad de tener que esperar a que el resto de compañeros lo concluyera; con lo que se logró respetar el ritmo de aprendizaje particular de cada uno de los estudiantes.

La propuesta estaba organizada de tal manera que un estudiante podía cubrir todos los niveles de la formación primaria en un plazo de cuatro años, aunque era totalmente factible que lo hicieran en menos tiempo. Algunos estudiantes, por el interés y la dedicación invertida, llegaron inclusive a concluir la formación primaria en dos años, como fue el caso José María Pilaguano o Alfonso Patango, dos de los educadores comunitarios de las escuelas indígenas, y que hasta el día de hoy siguen dando clase en una escuela de la comunidad de Guayama.

El plazo de cuatro años como tiempo máximo para concluir la formación primaria no fue una decisión al azar, se la tomó "tras realizar un estudio en el que se vio que su realidad socioeconómica y migratoria no permitía a los niños indígenas acudir a las escuelas por más de cuatro años, por lo que la mayoría no concluía y no recibían su titulación de primaria, lo cual les limitaba enormemente" (Martínez 2004, 257).

Para finalizar la primaria los estudiantes que aprobaban todos los niveles debían tomar un curso intensivo de nivelación, que, como su nombre lo indica, tenía como objetivo nivelar a los estudiantes en aquellos conocimientos y destrezas que quedaron un poco débiles durante el proceso formativo. Normalmente, el curso se desarrollaba durante agosto y septiembre, en una de las escuelas del sector. Debido a que no 
eran muchos los estudiantes que concluían cada año todos los niveles de formación, el curso de nivelación se organizaba para todos los estudiantes que habían concluido los niveles, sin importar la escuela en la que habían estudiado. ${ }^{35}$

Con el objetivo de aprovechar al máximo el tiempo y cumplir con el objetivo de la nivelación, el curso funcionaba con la modalidad de internado: los estudiantes permanecían en el centro de lunes a viernes, y regresaban a sus comunidades los fines de semana. En cuanto a la organización del curso de nivelación, Íñiguez y Guerrero nos relatan lo siguiente:

Normalmente el primer mes es de estudios intensos: de 7 a 12 y de 14 a 18h00; la noche se aprovecha para dialogar con los niños sobre los problemas de su comunidad y de su pueblo. El segundo mes se alterna estudio con trabajo. Por la mañana estudio y por la tarde talleres de sastrería, carpintería, zapatería u otro tipo de artesanías.

Se intenta completar el currículum del nivel primario impartiendo clases de las cuatro áreas fundamentales: matemáticas, idioma nacional, ciencias naturales y ciencias sociales.

Si bien todo el proceso educativo está orientado para que el niño indígena pierda el miedo a expresar su vivencia cultural, en el curso de nivelación se logra hacer realidad, por medio de cantos, teatro, juegos, adivinanzas, cuentos y algunas experiencias vividas en sus comunidades.

Otra de las intenciones del curso es que el niño o joven indígena pueda responsabilizarse de la continuidad de su proceso educativo. Para esto se le propone algunas alternativas de trabajos grupales. $(1986,102)$

A diferencia del trabajo diario en la escuela, que estaba en manos de un educador indígena, el curso de nivelación era responsabilidad de jóvenes voluntarios que viajaban a Zumbahua en los meses de vacaciones para apoyar a los misioneros, siempre con la colaboración de un docente indígena que tenía como función ayudar a que los estudiantes entendieran al voluntario que, por lo general, no hablaba kichwa.

Haciendo un recuento, podemos decir que se trata de una propuesta educativa que se nutre de muchos de los postulados de la educación popular en boga en dicha época, que tienen su raíz en los planteamientos de Paulo Freire y en su propuesta de la pedagogía liberadora. Si 
esto era cierto para la propuesta educativa macro, también lo era para las propuestas de cada una de las subáreas: en la enseñanza de la lectura y escritura, por ejemplo, encontramos varios elementos de la metodología de alfabetización freiriana. Lo mismo ocurre en la subárea de ciencias sociales, pues en las directrices metodológicas encontramos una fuerte influencia del método "ver, juzgar y actuar" de monseñor Leonidas Proaño (Arrobo 2007).

Al valorar desde la discusión teórica generada en Ecuador y América Latina en torno a la educación indígena, podemos afirmar que la propuesta educativa de las Escuelas Indígenas del Quilotoa fue un avance incipiente de lo que, una década después, en los 80 , se conocería como el modelo de educación intercultural bilingüe. A diferencia de la educación bilingüe de mantenimiento y desarrollo, cuya meta central era consolidar en los estudiantes el manejo de su lengua materna a la par que propiciar el aprendizaje de la lengua hegemónica, la EIB era un tipo de educación que asimismo se preocupaba por la dimensión cultural y que buscaba adecuarse a la cultura de referencia de los estudiantes y abrir la posibilidad para que aquellos se apropiaran de elementos de la cultura hegemónica. Luis Enrique López, experto en el tema, categoriza a la EIB de la siguiente manera:

La EIB es [...] una educación enraizada en la cultura de referencia inmediata de los educandos pero abierta a la incorporación de elementos y contenidos provenientes de otros horizontes culturales, incluida la propia cultura universal. Es también una educación vehiculada en un idioma amerindio y en castellano o portugués que propicia el desarrollo de la competencia comunicativa de los educandos en dos idiomas a la vez: el materno y uno segundo.

La denominación de intercultural está referida explícitamente a la dimensión cultural del proceso educativo y a un aprendizaje significativo y social y culturalmente situado; así como también a un aprendizaje que busca responder a las necesidades básicas de los educandos provenientes de sociedades étnica y culturalmente diferenciadas.

La dimensión intercultural de la educación está también referida tanto a la relación curricular que se establece entre los saberes, conocimientos y valores propios o apropiados por las sociedades indígenas y aquellos desconocidos y ajenos cuanto a la búsqueda de un diálogo y de una 
complementariedad permanentes entre la cultura tradicional y aquella de corte occidental, en aras de la satisfacción de las necesidades de la población indígena y de mejores condiciones de vida. (López 2001, 17-8)

Ahora bien, si algo diferencia a la propuesta educativa de las Escuelas Indígenas del Quilotoa de las propuestas de EIB es su sentido, pues tanto la apuesta por el fortalecimiento de la lengua y la cultura propia como la apuesta por la apropiación del español y ciertos elementos de la cultura blanco-mestiza tenían como finalidad su reconstitución como pueblo y la construcción de un nuevo tipo de relación con la sociedad mayor. En esa medida, se trata de una propuesta que rebasó el horizonte estrictamente lingüístico y culturalista en el que muchas veces se han quedado las iniciativas de EIB en la región, en especial aquellas impulsadas desde el Estado.

\section{Los educadores comunitarios y su formación}

Como vimos arriba los educadores de las escuelas indígenas eran comuneros a los que el equipo de apoyo de las escuelas, previa sugerencia de la comunidad, les solicitaba hacerse cargo de la escuela. Hubo casos, aunque los menos, en que los comuneros se autopostularon para ser educadores de la escuela de su comunidad o de otra comunidad.

Los comuneros tenían que cumplir con ciertos requisitos básicos para pasar a ser educadores de las escuelas, siendo las principales el que contaran con un nivel básico de formación formal (preferiblemente si habían concluido con la educación primaria), que hablaran kichwa, así como cierta trayectoria de trabajo a nivel de la organización y reconocimiento por parte de la comunidad. ${ }^{36}$

Una vez en el campo de acción, los educadores comunitarios tenían que cumplir un sinnúmero de actividades y propender a cumplir el perfil esperado por los comuneros que consideraba los siguientes atributos y roles:

36 Alfonso Patango, entrevista personal, 8 de mayo de 2014, comunidad de Guayama San Pedro, parroquia Chugchilán, cantón Sigchos. 
- Ser respetuoso de las decisiones de los padres de familia y dirigentes,

- Deben ser responsables en el trabajo asignado,

- Hablar correctamente el kichwa,

- Impulsar el desarrollo de la comunidad,

- Residir en la comuna, en lo posible ser miembro de ella,

- Ser conocedor de los problemas de la comuna. (Manangón, Baltazar y Trávez 1992, 67)

La figura del educador comunitario era de una importancia central en la experiencia educativa, pues uno de los postulados de la "educación propia" era que aquella tenía que ser llevada adelante por comuneros de la misma zona, que hubieran vivido lo que los demás habían vivido, que entendieran el contexto y los problemas de la comunidad, que apuntaran a sacarla adelante por medio de la educación y otros medios, y que - algo de suprema importancia para los comuneros - trataran bien y de manera respetuosa a los niños y los demás comuneros. En esa medida, la figura del educador comunitario era muy diferente y mucho más compleja que la figura del "profesor" de las escuelas fiscales de ese tiempo. Leonardo Cunuhay, uno de los primeros educadores comunitarios, explica con claridad la diferencia entre el rol de los educadores comunitarios y el rol de los profesores:

La diferencia que existe entre el educador y el profesor es que el término educador se utiliza desde la creación de la educación indígena, porque nuestra responsabilidad no es únicamente la de profesor, porque nosotros desarrollamos un nuevo tipo de relación pedagógica no simplemente con los niños sino también con todos los miembros de la comunidad, nuestra labor no se circunscribe a la instrucción solamente, sino al desarrollo integral.

Porque el educador no va la comunidad como un patrón o un sabio o sentirse superior a los demás, más bien va como un miembro de la comunidad a ayudar a solucionar ciertas problemáticas que existen dentro de la comunidad, por todo esto nos denominamos educadores comunitarios bilingües y no simplemente profesores bilingües.

El término profesor lo han denominado así porque la persona que va a trabajar en las comunidades solo va a profesar y piensa que él solo sabe, 
van con una ideología desfavorable se sienten patrones, sin dar importancia a la comunidad.

Al hablar el profesor es orgulloso, se siente superior a los demás, entonces la diferencia que está a la vista es que el educador se preocupa de los problemas de las comunidades y el profesor solo cumple con sus clases en la escuela. (1993)

El punto anterior se conecta con otro y tiene que ver con la jornada de trabajo. El rol del educador comunitario era un rol complejo e incluía varias funciones pues, además de hacerse cargo y velar por el proceso de enseñanza-aprendizaje de los niños y adolescentes, tenía bajo su responsabilidad el proceso de alfabetización de adultos y la organización de la comunidad, funciones que copaban las tres jornadas del día: diurna, vespertina y nocturna. Un día normal de trabajo de un educador se organizaba de la siguiente manera: en la mañana trabajaba con los niños/adolescentes, en la tarde con los adultos en la alfabetización, y en la noche se dedicaba a trabajar con la comunidad y la organización, liderando y animando los procesos. ${ }^{37}$

Lo dicho nos da una idea clara del carácter multifuncional del educador comunitario, a la vez que ayuda a visualizar el lugar central que aquel ocupaba dentro la comunidad. Y es que el educador comunitario, por las múltiples funciones que realizaba y por la cercanía con los comuneros, la mayoría de veces, terminaba siendo el verdadero líder de la comunidad y la persona de confianza de sus dirigentes. ${ }^{38}$ Esta suerte de multifuncionalidad del educador comunitario y de la escuela indígena constituía, sin lugar a dudas, una de las originalidades de las Escuelas Indígenas del Quilotoa.

En términos de formación, los educadores comunitarios se clasificaban en dos grupos: aquellos que habían concluido la primaria, y aquellos que tenían algunos años de estudio pero que no habían logrado concluirla, considerando que tanto los primeros como los segundos constituían el grupo con mayor nivel de escolaridad en la zona, pues como explicamos en la primera parte de este capítulo, en la década de

37 Mery Martínez, entrevista personal, 19 de junio de 2014, Latacunga.

38 Ibíd. 
los 70 la mayor parte de la población de las comunidades indígenas en las que se crearon las escuelas era analfabeta. Con esta realidad, el tema de la capacitación y formación de los docentes se convirtió en un asunto prioritario y en una política permanente.

El proceso de formación de los educadores fue por medio dos modalidades. Por un lado, estaban los cursos de capacitación para el trabajo en el aula; por otro, estaban los procesos de formación de largo aliento, que concluían con la titulación respectiva. Con relación a la primera modalidad, los educadores recibían entre ocho y nueve cursos cada año, con diferente duración, que tenían como objetivo fundamental reforzar sus habilidades para el trabajo en el aula y en la comunidad en general: planificación educativa, metodología, manejo del material didáctico, desarrollo comunitario, conservación de suelos, contabilidad, catequesis (SEIC 1989).

En algunas ocasiones los cursos eran organizados por el mismo equipo de apoyo de las escuelas, pero la mayoría de veces por otras instituciones del Estado y oenegés que trabajaban en la zona: el Ministerio de Salud, el Ministerio de Agricultura y Ganadería, FODERUMA, FEEP, SWISS Contact, las Escuelas Radiofónicas Populares de Riobamba y el Centro de Investigación sobre Educación Indígena, entre otras (SEIC 1989).

Respecto a la segunda modalidad, los educadores comunitarios se vieron permanentemente presionados, desde diferentes sectores, a continuar con su proceso de formación formal y a obtener las titulaciones básicas. Algunos educadores comunitarios se vieron ante la urgente necesidad de concluir la educación primaria, mientras que otros se volcaron a avanzar con el ciclo básico de la secundaria, para luego continuar con la formación de bachillerato y, finalmente, concluir con la educación superior.

En este proceso fueron clave ciertas instituciones que en el momento ofrecían los servicios educativos requeridos, en especial el Instituto Radiofónico Fe y Alegría (IRFEYAL) y el Instituto Pedagógico Intercultural Bilingüe Quilloac. Mientras en IRFEYAL los educadores estudiaron el ciclo básico de la secundaria, en el Instituto Pedagógico estudiaron 
el bachillerato y la especialización de profesor de instrucción primaria. ${ }^{39}$ Para su formación a nivel de licenciatura los educadores optaron por dos vías: algunos por el Programa Académico Cotopaxi, programa de licenciatura en EIB ofertado por la UPS, con sede en Zumbahua, y otro de licenciatura en Educación ofertada por Universidad Técnica de Babahoyo que tenía una extensión en Quito. ${ }^{40}$

El tema de la capacitación y formación de los educadores comunitarios era importante no solo porque era una condición básica - así lo consideraban los miembros del equipo de apoyo y los mismos educadorespara el mejoramiento del proceso educativo sino, además, por las críticas de las que las escuelas indígenas eran blanco. Una de las más recurrentes por parte de la población mestiza y, de manera especial, por parte de los docentes de las escuelas fiscales, era que los educadores de las escuelas indígenas no estaban preparados para la docencia, y que ni siquiera contaban con título de primaria. En este sentido, la formación constituía una estrategia de primera mano para construir la legitimidad de las escuelas frente a la población mestiza, pero también frente a los mismos comuneros que confiaban en las escuelas indígenas y apostaban por ellas. ${ }^{41}$

\section{El modelo de gestión y la participación de la comunidad}

Hasta aquí hemos analizado la propuesta educativa de las escuelas indígenas y la figura del educador comunitario, pero, ¿cómo operaba el tema de la gestión de las escuelas?, ¿qué relación había entre la escuela y la comunidad?, ¿cómo participaba la comunidad en su funcionamiento?

Un tema de reflexión recurrente dentro de los procesos de educación indígena en Ecuador y la Región Andina ha sido el de la participación y el control comunitario de la educación. Su importancia se debe a que la participación comunitaria ha sido vista como una de las condiciones clave

39 En 1991 se creó el colegio Jatari Unancha, como parte del mismo proyecto educativo impulsado por los salesianos y los líderes indígenas. Desde ese año en adelante, los estudiantes que se graduaban en las escuelas indígenas pasaban, por lo general, a realizar sus estudios de secundaria en dicho colegio.

40 Pedro Cunuhay, entrevista personal, 7 de mayo de 2014, comunidad de Chinaló, parroquia de Chugchilán, cantón Sigchos.

41 Padre Marcelo Farfán, entrevista personal, 10 de julio de 2014, Quito. 
para asegurar una educación con pertinencia social, cultural e inclusive política (López 2001). En contextos en los que no ha llegado el Estado o en los que este ha llegado con propuestas ajenas, la participación comunitaria ha sido una condición central de las iniciativas educativas propias, inclusive para sostener a las mismas escuelas. Este ha sido el caso, por ejemplo, de las escuelas zapatistas en la Selva Lacandona, México, o del proyecto educativo indígena impulsado por el CRIC, en el Valle del Cauca, Colombia. ${ }^{42}$

En el caso de la experiencia objeto de nuestro estudio, la participación de la comunidad fue siempre prioritaria, no solo para la creación y sostenimiento de las escuelas y del educador comunitario, sino asimismo para el seguimiento y direccionamiento del proceso educativo. Demos una mirada a algunos de estos puntos.

Una vez que se decidía iniciar con la experiencia educativa en un determinado territorio, la gestión de la comunidad de dicho territorio era fundamental, pues era la encargada de impulsar la creación de la escuela, proceso que implicaba desde la donación de un terreno individual o comunal hasta la construcción de la escuela y su equipamiento. Cierto es que la comunidad muchas veces contaba con apoyo financiero del equipo de apoyo de las escuelas y de ciertas fundaciones que trabajaban en la zona, pero también es cierto que la comunidad era la responsable —en última instancia - de la construcción. No está por demás decir que la comunidad también era la encargada de dar mantenimiento a la escuela cada cierto tiempo o cuando ameritaba por las condiciones climáticas adversas.

En la sistematización de las vivencias de los primeros educadores comunitarios, encontramos abundantes testimonios que dan cuenta de este tema, y de las dificultades que se tenían que sortear, pues no debemos olvidar que estamos hablando de zonas en las que, muchas veces, estaban

42 Para la experiencia de las escuelas zapatistas se puede revisar el texto de Bruno Baronet Autonomía y educación indígena: Las escuelas zapatistas de la Selva Lacandona de Chiapas (2012); mientras que para la experiencia del proyecto educativo impulsado por el CRIC se puede revisar ¿Qué pasaría si la escuela...? 30 años de construcción de una educación propia (Consejo Regional Indígena del Cauca 2004), y el texto de Johanne Rappaport El espacio del diálogo pluralista: Historia del Programa de Educación Bilingüe del Consejo Regional Indígena del Cauca (Rappaport 2006). 
en pleno proceso de lucha por la tierra, y en las que todo este proceso debía ser llevado adelante de manera clandestina y con escasos recursos. Como era de esperarse, el sistema de la minga fue sustancial tanto para la construcción de la escuela como para su mantenimiento.

Otro ámbito en el que la comunidad tenía un gran protagonismo era aquel relacionado con el educador comunitario. La comunidad era la responsable de seleccionar, de entre los comuneros, al educador que se haría cargo de la escuela, o en su defecto, de proponer posibles candidatos para dicho cargo. ${ }^{43}$ Como anotamos, uno de los principios fundamentales de las escuelas indígenas era que el educador debía ser de las mismas comunidades, condición básica —según los comunerosde un proyecto de educación propia.

El trabajo de la comunidad no concluía simplemente con la selección del educador, sino que incluso estaba bajo su responsabilidad contribuir con su subsistencia diaria y su seguimiento. Sobre el primer punto, si bien es cierto que el equipo de apoyo se ingeniaba para buscar recursos financieros para los educadores comunitarios, recursos que por lo general eran escasos, también es cierto que la comunidad debía contribuir a la manutención del educador, por cuanto dichos recursos no le alcanzaba para vivir. En algunas ocasiones las familias se turnaban para invitar a comer al educador en sus casas, otras veces las familias entregaban productos de sus huertas a los docentes para que se preparara su comida. Había ocasiones en que las familias se organizaban para entregar al educador cierto monto de dinero. Revisemos lo que dice Francisco Pastuña, uno de los primeros educadores comunitarios de la zona, cuando le preguntamos sobre cómo se sostenían en los primeros años de vida de las escuelas:

Gracias a los padres de familia que me apoyaron, en esa temporada nos regalaban cosas o ponían cinco sucres. Cinco sucres nos daban los padres de familia; era el aporte para el profesor. Nos daban papitas, traían cebollitas, oquita, habitas, eso me daban. Resto, yo compraba: manteca, arrocito, fideo, así. En ese entonces aportaban a cinco sucres. Con esito

43 Francisco Pastuña, entrevista personal, 18 de abril de 2014, comunidad del Quilotoa, parroquia Zumbahua, cantón Pujilí. 
yo compraba porque igual yo no tenía pues. Entonces, con eso yo defendía, yo entraba lunes y salía viernes, porque yo vivía lejos. La escuela era mi casa. Yo tenía que caminar tres horas, no podía ir a la casa todos los días, entonces, yo quedaba a dormir ahí en la escuela. ${ }^{44}$

Cuando el educador era de la misma comunidad, también se daban casos en que las familias se organizaban y turnaban para trabajar la parcela del educador, de tal manera que él y su familia pudieran contar con productos para el día a día. ${ }^{45}$

Sobre el segundo punto, el relacionado con el seguimiento, la comunidad era asimismo la responsable de asegurar que el educador cumpliera con sus responsabilidades. Para ello, en cada reunión o asamblea de la comunidad, el educador debía rendir cuentas ante los comuneros sobre lo que había trabajado en el aula, contemplando los avances logrados, pero también los problemas encontrados; luego de lo cual recibía sugerencias y retroalimentación por parte de los padres de familia. La comunidad, además, era la encargada de vigilar que el educador cumpliera con sus funciones extra clase que, como ya adelantamos, constituía un aspecto central del trabajo del educador. ${ }^{46}$

Hasta aquí en lo que al papel de la comunidad se refiere, pero, ¿cuál era el papel del equipo de apoyo? Este estaba conformado por miembros del equipo de la Pastoral y tenía bajo su responsabilidad varias actividades que podrían agruparse en tres grandes líneas de trabajo: el apoyo académico-pedagógico, la búsqueda de sostenibilidad financiera y la intermediación con las instituciones externas (Íñiguez y Guerrero 1986).

Con relación a la primera línea de trabajo, el equipo de apoyo era el encargado de orientar y asesorar a los educadores en términos de los contenidos a ser trabajados y la metodología a utilizar, elaborar — conjuntamente con los educadores - las cartillas y demás material didáctico para el trabajo en el aula, y organizar y realizar cursos de capacitación para los educadores. En cuanto al segundo punto, el equipo de apoyo era el encargado de buscar financiamiento tanto para las bonificaciones de

44 Ibíd.

45 Fernando Garcés, entrevista 21 de abril de 2015, por Skype.

46 Ibíd. 
los educadores comunitarios como para la elaboración de los materiales didácticos. Por último, la tercera línea de trabajo del equipo de apoyo consistía en cumplir el papel de intermediario entre las escuelas indígenas y los organismos del Estado y las oenegés.

Para cerrar este apartado es necesario dar una idea general de cómo se sostenían financieramente las escuelas indígenas en el período objeto de nuestro estudio (1975-1988), pues constituye un tema bastante sensible. El proyecto de las escuelas indígenas fue financiado por diferentes actores a lo largo de su primera etapa de vida, siendo el común denominador la ausencia de un financiamiento estable y adecuado (en términos de cantidad) por parte del Estado. Entre las principales instituciones que apoyaron a las escuelas indígenas están los voluntarios italianos Matto Grossos (que trabajaban conjuntamente con los salesianos en el equipo de la Pastoral), FODERUMA, la Fundación Interamericana, la Fundación Tierra de Hombres y el MEC, mediante la Dirección Provincial. El apoyo del MEC inició recién en 1980 y se concentró exclusivamente en bonificaciones para los educadores comunitarios, que nunca se acercaron, ni de lejos, al valor del sueldo básico del momento (Íñiguez y Guerrero 1986).

\section{El aporte de las Escuelas Indígenas del Quilotoa}

Hemos analizado el contexto en el que se crearon las Escuelas Indígenas del Quilotoa y su proceso de creación y consolidación. Hemos analizado también, y con cierto detalle, la propuesta educativa que las escuelas indígenas implementaron y su modelo de gestión. Para cerrar el capítulo es necesario discutir sobre el aporte de las escuelas indígenas a la zona en cuestión, pues solo de esta manera tendremos una idea clara sobre el nivel de pertinencia de la iniciativa educativa que nos interesa.

Para comenzar es necesario recuperar una de las ideas que planteamos en los acápites anteriores, a saber, aquella relacionada con la multifuncionalidad: la escuela indígena, además de ser un espacio de formación de las nuevas generaciones de las comunidades de la zona, fue una instancia alrededor de la cual la comunidad se organizaba para enfrentar los problemas y apuntalar la consecución de sus proyectos como colectividad, o, como dirían Martínez y Burbano, la escuela era, 
además, "un espacio y tiempo para analizar los problemas, organizar los trabajos, evaluar y proyectarse en función de los objetivos comunes” (Martínez y Burbano 1994, 80).

La reflexión anterior es relevante por cuanto nos invita a analizar el aporte de las escuelas indígenas en un sentido amplio: en tanto que instancia educativa, en el sentido estricto de la palabra, pero asimismo como espacio de organización y promoción de la comunidad.

\section{El aporte educativo}

En términos educativos el aporte de las Escuelas Indígenas del Quilotoa fue altamente positivo en el período objeto de nuestro análisis, pues no solo contribuyeron a educar a un porcentaje importante de la población de la zona en cuestión, sino que dicha formación fue funcional para el proyecto político de aquella. Revisemos algunos datos que contribuyen a fundamentar la afirmación anterior.

En primer lugar, tenemos que las escuelas indígenas contribuyeron a reducir de manera radical el nivel de analfabetismo de las comunidades en las que incidieron, donde — como vimos al inicio del capítulo- el analfabetismo afectaba a la mayor parte de la población. De acuerdo con Sánchez Parga (2005), en los sectores donde operaron las escuelas indígenas el porcentaje de analfabetismo se redujo a la mitad: de $72 \%$ en 1974 a $35 \%$ en $2001 .{ }^{47}$ Si comparamos estos datos con el nivel de reducción del analfabetismo en el resto de las zonas rurales de la provincia de Cotopaxi (de $45 \%$ en 1974 a $19 \%$ en 2001), vemos que la reducción en las zonas de las escuelas indígenas es un tanto menor; que es entendible si se considera que los sectores en los que operaban estas escuelas eran en los que se concentraba la población indígena, así como los mayores niveles de pobreza y extrema pobreza.

47 No contamos con datos sobre el nivel de analfabetismo en 1988, año en que se cerró el período que nos interesa para el análisis. Sin embargo, consideramos que los datos de 2000 nos dan una idea clara del ritmo y nivel con el que el analfabetismo se redujo en la zona por efecto de las escuelas indígenas, y nos permiten deducir que en 1988 pudieron ser parecidos. 
La reducción del analfabetismo constituía un proceso de suprema importancia en la zona pues, como se discutió arriba, el analfabetismo fue el detonante que llevó a la población indígena a movilizarse por la educación, tanto por sus implicaciones a nivel personal como colectivo.

Al comparar la reducción del analfabetismo por sexos, se puede constatar que, si bien el nivel de reducción del analfabetismo fue mayor en la población masculina, fue también importante en la población femenina: $-26,4 \%$ entre 1974 y 1990 para el caso de los hombres, y -23,3 \% para el caso de las mujeres (Sánchez Parga 2005). Los datos sobre la reducción del nivel de analfabetismo por sexo son supremamente interesantes por cuanto nos permiten visibilizar otro de los grandes logros de las escuelas indígenas, a saber, la escolarización de las mujeres indígenas.

En el segundo acápite se hizo alusión a la importancia que el equipo de apoyo y los líderes indígenas, aunque no los comuneros llanos, asignaban al proceso de formación de las mujeres de la zona, y a las estrategias, muchas de ellas anecdóticas, que se implementaron para asegurar que los padres de familia enviaran a sus hijas mujeres a la escuela. Y es que para el equipo de apoyo y los líderes indígenas la formación de las mujeres era supremamente importante por cuanto, desde su punto de vista, ellas tenían un papel central en la reproducción y el mantenimiento de la cultura en la zona (Martínez y Burbano 1994).

Otro indicador interesante que debe ser considerado es el nivel de instrucción. Al comparar la situación entre 1974 y 2000, se puede constatar, además, una reducción importante del porcentaje de personas sin ninguna instrucción en la zona en la que operaron las escuelas indígenas: de 66 \% en 1974 a 37,8 \% en 2000 (Martínez y Burbano 1994), aspecto que también da cuenta del impacto positivo de las escuelas indígenas. Y es que, como sostiene Carmen Martínez, el mayor aporte de las escuelas indígenas no solo fue el haber brindado un servicio educativo tolerante y pertinente con el contexto, sino y sobre todo, el haber llegado a zonas a las que nunca antes había llegado ningún servicio educativo (2004).

Además de haber contribuido a ampliar el porcentaje de población indígena alfabeta y con algún nivel de instrucción, las escuelas indígenas jugaron un papel fundamental en la formación de ciertas habilidades y 
actitudes clave para el desarrollo económico, político y cultural de las comunidades de la zona, aspectos que fueron valorados, desde un inicio, tanto por los líderes indígenas como por el equipo de apoyo. Nos referimos a las habilidades y actitudes vinculadas al tema productivo pero, además, al tema del respeto de la cultura propia. El trabajo de Martínez y Burbano nos es de utilidad para sustentar esta parte.

Rodrigo Martínez y Bolívar Burbano realizaron, entre 1992 y 1993, un estudio comparativo sobre el impacto de tres experiencias de educación indígena que operaron en las zonas rurales de la provincia de Cotopaxi en las décadas 80 y 90: el Proyecto de Educación Bilingüe Intercultural financiado por la Cooperación Alemana, el Proyecto de las escuelas de la DIPEIB-C, y el Proyecto de las Escuelas Indígenas del Quilotoa. ${ }^{48} \mathrm{El}$ estudio analizó el impacto que los tres proyectos tuvieron en varias esferas de la vida de niños y niñas involucrados en el proceso educativo, siendo las más importantes, desde nuestro punto de vista, aquellas relacionadas con el tema del manejo de la lengua, el respeto del ámbito de la cultura propia y la preparación para el trabajo agrícola y la crianza de animales.

De acuerdo con el estudio en cuestión, las Escuelas Indígenas del Quilotoa, y muchas veces con una gran diferencia en comparación con las escuelas de los otros dos proyectos, fueron las que más aportaron para que niños y niñas de la zona se comunicaran en kichwa sin importar el contexto, y a que lo hicieran sin sentir vergüenza. Lo que tiene sentido y encuentra su explicación tanto en el hecho de que los educadores comunitarios fueron siempre indígenas de la zona, cuya lengua materna era el kichwa, como en el hecho de que la consigna de las escuelas indígenas era que todo el proceso educativo debía realizarse en la lengua materna.

El que niños y niñas se habituaran a comunicarse en kichwa y sin tener vergüenza alguna puede sonar como un tema un tanto trivial, pero no lo es: en primer lugar, porque en ese tiempo el kichwa era la lengua materna de la población infantil de la zona, la lengua en la que aprendían a comunicarse y mediante la cual aprendían a procesar el mundo;

48 El estudio, que se llama La educación como identificación cultural y la experiencia de educación indígena en Cotopaxi, fue publicado por Abya-Yala y el SEIC en 1994. 
y en segundo lugar, porque la lengua materna constituye un elemento fundamental de toda cultura y porque ha sido una de las reivindicaciones más sentidas de las organizaciones indígenas del país y la región.

De igual manera, las escuelas indígenas fueron las que más incentivaron en niños y niñas el respeto por las costumbres locales, el aprecio por sus productos, frente a la avalancha cada vez mayor de productos externos, y la participación en las mingas, una de las instituciones clave de la vida comunitaria y que constituía el soporte y la condición de posibilidad de varias de las prácticas de la vida cotidiana e iniciativas de la comunidad como la cosecha, la construcción de un estanque y la construcción de la escuela, entre otras.

Para los líderes indígenas y el equipo de apoyo las actitudes descritas eran fundamentales, pues constituían elementos centrales del proyecto de fortalecimiento del ámbito de la cultura propia de la zona (Bonfil 1991), proyecto al que también debían contribuir a alimentar las escuelas indígenas.

Por último, tenemos que las Escuelas Indígenas del Quilotoa, en comparación con las escuelas de los otros dos proyectos, fueron las que más contribuyeron a que niños y niñas aprendieran a trabajar la tierra y cuidar los animales, actividades centrales, aunque no las únicas, para el sostenimiento de las familias indígenas. En cuanto a esto último, vale recordar el hecho de que la propuesta de estudios de las escuelas indígenas contemplaba un área de actividades productivas orientada a preparar a los niños para el trabajo en el campo y para la producción de diferentes tipos de artesanía.

Para cerrar este punto conviene destacar el aporte de las escuelas indígenas al desarrollo de las comunidades por medio de sus graduados y sus acciones desarrolladas, que es una manera de evaluar el aporte de cualquier iniciativa educativa. En el caso de las escuelas indígenas tenemos que una buena parte de sus graduados pasaron a cumplir un papel protagónico en sus respectivas comunidades, ya sea como dirigentes, educadores, promotores de salud, catequistas, pero también impulsando proyectos de orden productivo. Revisemos lo que dicen tres de los primeros educadores comunitarios del proyecto en cuanto a estos aportes: 
La juventud de esta comunidad que ha estudiado en esta escuela está prestando servicio en la comuna como educadores comunitarios, alfabetizadores, catequistas, promotores de salud, dirigentes, etc. (Íñiguez y Guerrero 1986, 86)

Con los exalumnos hemos formado una pequeña organización, hemos conseguido un horno para hacer pan. Hemos iniciado la comercialización, pagando precios justos. (Sistema de Escuelas Indígenas de Cotopaxi 1989, 99)

Se ha formado una organización con los alumnos que han terminado la primaria. Varios son educadores comunitarios, promotores de salud, administradores de pequeñas tiendas comunales y encargados de pequeños proyectos productivos. (99)

Escuchemos lo que los mismos exalumnos de las escuelas comentan sobre el tema:

Soy promotor de salud, trabajo cuidando de las enfermedades, educando un poco a la gente. Yo trabajo también como gerente de comercialización viendo las tiendas [...] antes robaban las libras, pagaban menos, ahorita nosotros pagamos precios fijos y libra fija es bueno para adelantar más nuestra comunidad. (Íñiguez y Guerrero 1986, 115)

Yo trabajo en carpintería. Hacemos para la misma comunidad. Hacemos mesas, bancas, puertas, ataúd, todo, todo lo que piden. También estamos colaborando con otras comunidades y a los que más necesidad tienen de nosotros. (115)

Es que un porcentaje importante de los estudiantes que se graduaron en las escuelas indígenas, por el mismo hecho de contar con un nivel básico de formación formal, pasaron a ser personas valoradas en sus respectivas comunidades, y a las que - con el tiempo- la comunidad y la organización les delegó cada vez más responsabilidades y funciones.

En la misma línea de reflexión vale destacar el aporte de las escuelas indígenas al desarrollo y fortalecimiento de las organizaciones de segundo grado y al MICC, aspecto que ha sido visibilizado de manera insistente por los líderes de la zona y educadores comunitarios, pero también por estudiosos del tema como Carmen Martínez (2004) y José Sánchez Parga (2005). De acuerdo a este último, el 58 \% de los líderes 
del MICC, entre 1982 y 2002, se graduaron en las escuelas indígenas o estuvieron vinculadas a ellas en calidad de educadores comunitarios, lo que da cuenta del aporte de las escuelas en la formación de cuadros políticos, muchos de los cuales siguen vinculados a las organizaciones indígenas y a Pachakutik hasta el día de hoy.

\section{El aporte a la organización y desarrollo de las comunidades}

Hasta aquí lo que tiene que ver con el aporte educativo de las escuelas indígenas. Queda pendiente, sin embargo, revisar el otro lado de la moneda, aquel que hemos catalogado como el impacto de las escuelas en tanto que instancia de organización y promoción de la comunidad. En esta línea el aporte de las escuelas al desarrollo de la zona ha sido supremamente importante. Desde un inicio las escuelas se convirtieron en espacios clave en los que la población se encontraba para, con la intermediación y animación del educador comunitario, conversar sobre los problemas que les aquejaba, perfilar propuestas de acción y llevarlas a la práctica. Leamos lo que los educadores comunitarios planteaban en 1988 sobre el tema:

Tenemos una idea aproximada sobre la realidad que vivía el pueblo indígena hace unos quince años, gracias a lo que nos han contado algunos educadores comunitarios. En esta realidad, el servicio educativo fue cobrando valor en la medida en que ayudaba a organizarse para unir cabezas, sentimientos y trabajos e ir solucionando problemas fundamentales que afectaban a la mayoría.

La educación, en ese caso, se orienta al fortalecimiento de la organización, de la unidad, la solidaridad en situaciones difíciles, el trabajo común con la participación de la mayoría para resolver problemas que van en contra de la dignidad de la persona. Y es así como se va desarrollando la conciencia de que "valemos", de que no estamos solos, que somos un solo pueblo indígena, que nos unen los mismos problemas y por lo tanto tenemos que unirnos. (Sistema de Escuelas Indígenas de Cotopaxi 1989, 96)

El papel de la escuela varió dependiendo de la trayectoria histórica y situación del momento de las comunidades. En las comunidades donde no había aún organización, la escuela fue su base misma. En otras, en las que 
ya había cierto nivel de organización, la escuela fue el elemento que detonó el trabajo colectivo en procura de ciertos objetivos. En aquellas comunidades que vivían todavía enmarcadas por el régimen de hacienda, la escuela fue la instancia desde la que se organizó la lucha por la tierra. En otras, la escuela se terminó convirtiendo en un contrapoder de los cabecillas.

En todos los casos, sin embargo, la escuela fue un espacio central en la vida de la comunidad, desde donde se impulsaron varios de los proyectos más sentidos de la comunidad. Al referirse al tema en cuestión, uno de los primeros educadores comunitarios de Chaupi sostiene lo siguiente:

La Escuela Indígena fue la base de la organización, los adelantos que se lograron fueron:

- Se formó una organización con los alumnos que terminaron la primaria. Varios son educadores comunitarios, promotores de salud, administradores de pequeñas tiendas comunales y encargados de pequeños proyectos productivos.

- Se logró que la comuna se organice y se recuperaron las tierras que estaban perdidas para la mayor parte de comuneros.

- Hemos logrado recuperar lo más que es nuestro idioma y nuestra cultura.

- Mediante la Escuela hemos llegado a tener casa comunal, escuela, tienda comunal, cuyera, agua entubada, etc. (SEIC 1989,99)

Un educador comunitario de la escuela de la comunidad de San Francisco de Sarahuasi, por su parte, dice:

La comuna de San Francisco de Sarahuasi ha realizado los trabajos a base de la organización de las Escuelas Indígenas de Cotopaxi y se ha logrado:

- Construir la capilla para la santa misa.

- Construir un aula y un cuarto para la cocina de la escuela.

- Hemos logrado el agua entubada para la comuna.

- Se consiguió un pedazo de terreno para el huerto escolar.

- Los comuneros se organizaron para sacar la mora al mercado y evitar que los comerciantes les exploten pagando apenas 50 sucres por libra. 
- Ahora los comuneros quieren solicitar ayuda para tener de una vez la carretera. (SEIC 1989, 101-2)

Estos aportes positivos (tanto educativos como políticos) no deben hacernos pensar que no hubo problemas en el desarrollo de las escuelas y su proyecto educativo. De hecho, se dieron problemas que muchas veces provocaron la clausura de alguna de las escuelas o la pérdida de apoyo de las comunidades indígenas. Sin embargo, estamos de acuerdo con Carmen Martínez (2004) y Sánchez Parga (2005) en que, a pesar de los problemas y limitaciones, el aporte de las escuelas en la zona fue de una importancia única, tanto así que mucho de lo que aconteció en aquella en las tres últimas décadas no se hubiera dado sin su presencia o se hubiera dado de manera muy distinta.

Si recuperamos todo lo discutido en el presente capítulo y lo interpretamos a la luz de la perspectiva de la teoría del control cultural (Bonfil 1991), podemos concluir afirmando que, frente a la desatención del Estado y a la necesidad de formar a sus miembros en el marco de su proyecto de etnodesarrollo, las comunidades indígenas de la zona optaron por una estrategia de creación e innovación en materia educativa que concluyó con la construcción de una red de escuelas, que contribuyó no solo a fortalecer su matriz cultural sino también a alimentar su proyecto de etnodesarrollo. Decimos "innovación" y no solo "creación" por cuanto la creación de las escuelas fue de la mano de la generación de una propuesta educativa original que se adecuó a las características de la población de la zona y que respondió a sus problemas y aspiraciones.

La experiencia en cuestión marca una diferencia importante con otras experiencias de educación indígena desarrolladas en el país y la región, pues a diferencia de estas últimas, en las que la estrategia central fue la apropiación y refuncionalización de las escuelas y los proyectos educativos existentes, en la experiencia objeto de nuestro estudio la estrategia prioritaria fue la creación de escuelas y de un proyecto educativo novedoso, pues en la mayor parte de comunidades de la zona objeto de nuestro estudio simplemente no había escuelas y no llegaba la educación del Estado. 